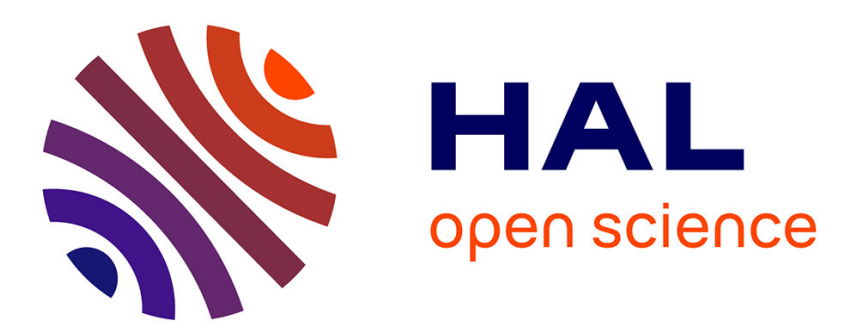

\title{
Sensorineural hearing loss impairs sensitivity but spares temporal integration for detection of frequency modulation
}

\author{
Nicolas Wallaert, Léo Varnet, Brian C.J. Moore, Christian Lorenzi
}

\section{To cite this version:}

Nicolas Wallaert, Léo Varnet, Brian C.J. Moore, Christian Lorenzi. Sensorineural hearing loss impairs sensitivity but spares temporal integration for detection of frequency modulation. Journal of the Acoustical Society of America, 2018, 144 (2), pp.720-733. 10.1121/1.5049364 . hal-02993063

\section{HAL Id: hal-02993063 https://hal.science/hal-02993063}

Submitted on 6 Nov 2020

HAL is a multi-disciplinary open access archive for the deposit and dissemination of scientific research documents, whether they are published or not. The documents may come from teaching and research institutions in France or abroad, or from public or private research centers.
L'archive ouverte pluridisciplinaire HAL, est destinée au dépôt et à la diffusion de documents scientifiques de niveau recherche, publiés ou non, émanant des établissements d'enseignement et de recherche français ou étrangers, des laboratoires publics ou privés. 


\title{
Sensorineural hearing loss impairs sensitivity but spares temporal integration for detection of frequency modulation
}

\author{
Nicolas Wallaert ${ }^{\mathrm{a})}$ and Léo Varnet \\ Laboratoire des Systèmes Perceptifs, Département d'Études Cognitives, Ecole Normale Supérieure, \\ Université Paris Sciences \& Lettres, Centre National de la Recherche Scientifique, 75005 Paris, France \\ Brian C. J. Moore \\ Department of Experimental Psychology, University of Cambridge, Downing Street, Cambridge CB2 3EB, \\ United Kingdom \\ Christian Lorenzi \\ Laboratoire des Systèmes Perceptifs, Département d'Études Cognitives, Ecole Normale Supérieure, \\ Université Paris Sciences \& Lettres, Centre National de la Recherche Scientifique, 75005 Paris, France
}

(Received 27 October 2017; revised 28 June 2018; accepted 20 July 2018; published online 15 August 2018)

The effect of the number of modulation cycles $(N)$ on frequency-modulation (FM) detection thresholds (FMDTs) was measured with and without interfering amplitude modulation (AM) for hearing-impaired (HI) listeners, using a 500-Hz sinusoidal carrier and FM rates of 2 and $20 \mathrm{~Hz}$. The data were compared with FMDTs for normal-hearing (NH) listeners and AM detection thresholds (AMDTs) for NH and HI listeners [Wallaert, Moore, and Lorenzi (2016). J. Acoust. Soc. 139, 3088-3096; Wallaert, Moore, Ewert, and Lorenzi (2017). J. Acoust. Soc. 141, 971-980]. FMDTs were higher for HI than for NH listeners, but the effect of increasing $N$ was similar across groups. In contrast, AMDTs were lower and the effect of increasing $N$ was greater for HI listeners than for NH listeners. A model of temporal-envelope processing based on a modulation filter-bank and a template-matching decision strategy accounted better for the FMDTs at $20 \mathrm{~Hz}$ than at $2 \mathrm{~Hz}$ for young NH listeners and predicted greater temporal integration of FM than observed for all groups. These results suggest that different mechanisms underlie AM and FM detection at low rates and that hearing loss impairs FM-detection mechanisms, but preserves the memory and decision processes responsible for temporal integration of FM. (C) 2018 Acoustical Society of America. https://doi.org/10.1121/1.5049364

[ICB]

Pages: $720-733$

\section{INTRODUCTION}

Communication sounds, including speech and animal vocalizations, contain relatively slow $(<5-10 \mathrm{~Hz})$ patterns of frequency modulation (FM) that play an important role in the discrimination and identification of the sounds (e.g., Rees and Malmierca, 2005; Zeng et al., 2005). Consistent with this idea, several psychophysical studies have shown that sensitivity to slow FM is associated with speech identification for both normal-hearing $(\mathrm{NH})$ and hearing-impaired (HI) individuals when speech reception is limited by acoustic degradation or competing speech sounds (Buss et al., 2004; Strelcyk and Dau, 2009; Johannesen et al., 2016). To understand the nature of the link between FM detection and speech perception, it is important to clarify the sensory and non-sensory mechanisms involved in FM detection and the effects of cochlear hearing loss and age on FM perception (Paraouty et al., 2016; Whiteford et al., 2017). That is the goal of the present study.

Many studies indicate that sensorineural hearing loss impairs the ability to detect FM, sometimes substantially (for a review, see Moore, 2014). This has been reported for a

${ }^{a)}$ Electronic mail: nicolas.wallaert@ens.fr wide range of audiometric configurations, stimulus levels, carrier frequencies, modulation rates and modulation patterns (e.g., Moore and Skrodzka, 2002; Lacher-Fougère and Demany, 1998; Buss et al., 2004; Strelcyk and Dau, 2009; Ernst and Moore, 2012; Johannesen et al., 2016; Kortlang et al., 2016; Paraouty et al., 2016). The deficits for hearingimpaired (HI) listeners are generally interpreted as resulting from degradation of the sensory encoding of FM. In the normal auditory system, information about FM is potentially conveyed by two cues: (1) temporal-envelope cues, related to the dynamic changes in the distribution of excitation along the basilar membrane within the cochlea (resulting in fluctuations in firing rate of neurons with characteristic frequencies below and above the carrier frequency); (2) temporal fine-structure (TFS) cues related to the waveform of the stimulus at each point on the basilar membrane (and the corresponding fine timing of the action potentials in neurons with different characteristic frequencies) (Rees and Malmierca, 2005; Paraouty et al., 2016). It has been proposed that for normal-hearing $(\mathrm{NH})$ listeners, FM is detected via TFS cues when the FM rate is low $(<5 \mathrm{~Hz})$ and when the carrier frequency is below about $4 \mathrm{kHz}$ (Demany and Semal, 1986, 1989; Sek and Moore, 1995; Moore and Sek, 1996; Moore and Skrodzka, 2002; Ernst and Moore, 2010; 
Paraouty et al., 2016; Paraouty et al., 2018; Wallaert et al., 2016), and via temporal-envelope cues when the FM is fast or when the carrier frequency is higher than $4 \mathrm{kHz}$ (Zwicker, 1956; Maiwald, 1967; Saberi and Hafter, 1995; Moore and Sek, 1996; Ernst and Moore, 2010; Paraouty et al., 2016; Paraouty et al., 2018).

The ability to use temporal-envelope cues may be reduced in the impaired auditory system because of (1) reduced conversion of FM into temporal-envelope cues at the outputs of the cochlear filters, resulting from the loss of outer hair cells in the cochlea and the consequent broadening of the filters (Glasberg and Moore, 1986); (2) loss of auditory-nerve fibers (Kujawa and Liberman, 2009) and the consequent impoverished (i.e., more noisy) neural representation of temporal-envelope cues in the early auditory system, as suggested by the psychophysical, electrophysiological and modeling work of Bharadwaj et al. (2015), Goodman et al. (2017), and Paul et al. (2017); (3) central changes, such as a loss of inhibition, which may affect envelope extraction and processing (Davis et al., 2010).

A reduced ability to use TFS cues may result from: (1) reduced precision of neural phase locking or a reduced number of neurons conveying phase locking (Kujawa and Liberman, 2009); (2) changes in the relative phase of response at different points along the basilar membrane (Ruggero, 1994), which would disrupt central mechanisms decoding TFS information based on correlation of the outputs of adjacent places (Shamma, 1985; Carney et al., 2002); (3) more complex and more rapidly varying neural TFS resulting from broader cochlear filters (Moore, 2008); (4) a mismatch between neural TFS information and the place on the basilar membrane that would "normally" respond to that information (Henry et al., 2016); (5) central changes following cochlear hearing loss, such as loss of neural inhibition (Moore, 2008), as suggested by models assuming that TFS may be recoded spatially at the brainstem level via lateral inhibition (Shamma and Lorenzi, 2013; see also Davis et al., 2010).

For HI listeners, the poorer-than-normal sensitivity to high-rate FM may result mainly from reduced conversion of FM into temporal-envelope cues, whereas the poorer-thannormal sensitivity to low-rate FM for low carrier frequencies may result from abnormal encoding and/or use of TFS cues. However, the origin of the observed deficits in FM detection remains a matter of debate (Ernst and Moore, 2012; Sheft et al., 2012; Kortlang et al., 2016; Paraouty et al., 2016; Wallaert et al., 2016; Whiteford et al., 2017), and it is possible that the deficits result partly from reduced "processing efficiency," that is a reduced ability of the central auditory system to make optimal use of available sensory information, perhaps caused by a decline in short-term memory and decision-making capacities. Consistent with this idea, recent work suggests that aging and sensorineural hearing loss are associated with reduced processing efficiency (modeled as increased "internal noise") for amplitude-modulation (AM) detection (Füllgrabe et al., 2015; Paraouty et al., 2016; Wallaert et al., 2017), although some studies have shown no relationship between age and sensitivity to AM (Schoof and Rosen, 2014, Whiteford et al., 2017).
The present study was designed to clarify the relative contributions of sensory and non-sensory factors to FM detection for older $\mathrm{HI}$ listeners by combining a psychophysical and modeling approach. To do this, temporal integration was compared for AM and FM detection. Temporal integration here refers to the improvement in modulation detection thresholds with increasing number of modulation cycles, $N$ (Viemeister, 1979; Hartmann and Klein, 1980). The temporal integration of AM and FM is assumed to reflect central (i.e., decision) processes involving "multiple looks" (Sheft and Yost, 1990; Viemeister and Wakefield, 1991) or a template-matching process (Dau et al., 1997a,b).

Wallaert et al. (2017) measured AM detection thresholds (AMDTs) for $\mathrm{NH}$ listeners and listeners with mildto-moderate sensorineural hearing loss using a carrier frequency of $500 \mathrm{~Hz}$ and modulation rates of 2 and $20 \mathrm{~Hz}$. AMDTs decreased (i.e., improved) with increasing $N$ for both groups and AMDTs were smaller for the HI than for the $\mathrm{NH}$ group. Also, temporal integration of AM (the beneficial effect of increasing $N$ ) was greater for the HI group. Wallaert et al. (2017) developed a computational model based on the modulation-filterbank concept and a templatematching decision strategy (Dau et al., 1997a,b) to account for the data. The data and the simulations suggested that the loss of amplitude compression in the impaired cochlea was mainly responsible for the enhanced sensitivity and temporal integration of AM found for the HI listeners. They also suggested that, for AM detection, cochlear damage is associated with increased internal noise, but preserved short-term memory and decision mechanisms. We thus reasoned that, if FM detection relies on the use of temporal-envelope cues resulting from FM-to-AM conversion at the outputs of the cochlear filters, sensorineural hearing loss should have similar effects on the temporal integration of AM and FM cues. In other words, temporal integration of FM should be greater for $\mathrm{HI}$ listeners than for $\mathrm{NH}$ listeners.

In the present study, FM detection thresholds (FMDTs) were measured at $40 \mathrm{~dB}$ sensation level (SL) for listeners (aged between 50 and 64 years) with mild-to-moderate sensorineural hearing loss (designated HIo), using a $500-\mathrm{Hz}$ sinusoidal carrier and modulation rates of 2 and $20 \mathrm{~Hz}$. For HIo listeners with absolute thresholds of $40 \mathrm{~dB} \mathrm{HL}$ or more, the level was set to $80 \mathrm{~dB}$ sound pressure level (SPL). FMDTs were measured with and without interfering AM at the same rate as the FM. The AM was present in both intervals of a forced-choice trial, and was therefore uninformative, whereas the FM was present in only one interval. The task was to identify the interval with the FM. The AM was used to make it more difficult for the listeners to use temporal-envelope cues. In particular, if FM is detected by monitoring changes in excitation at a single point on the excitation pattern (Zwicker, 1952, 1956), the interfering AM should greatly impair performance (Moore and Sek, 1996). FMDTs were also measured with and without interfering $\mathrm{AM}$ for young $\mathrm{NH}(\mathrm{NHy})$ listeners and older $\mathrm{NH}(\mathrm{NHo})$ listeners matched in age with the HIo listeners. The data were compared with FMDTs measured by Wallaert et al. (2016) for NHy and NHo listeners and AMDTs measured by Wallaert et al. (2016) and Wallaert et al. (2017) for NHy, 
NHo, and HIo listeners under identical conditions. Finally, the data were compared to the predictions of a model of temporal-envelope processing based on the assumption that modulation detection is achieved by cross-correlating the outputs of AM channels with memory-stored templates according to an "optimal detector" scheme (Dau et al., 1997a,b; see also Ardoint et al., 2008). The model incorporated stages simulating temporal-envelope processing by low-level sensory mechanisms (cochlear filtering, instantaneous amplitude compression, adaptation), mid-level processes (bandpass AM filtering), and higher-level non-sensory processes (internal noise, memory decay in the temporalenvelope domain, and template matching).

\section{METHOD}

\section{A. Listeners}

\section{Hearing-impaired listeners}

Twelve HIo listeners aged between 50 and 64 years [mean $=59$ years; standard deviation $(\mathrm{SD})=4$ years $]$ were tested for FM detection with interfering AM. Nine of these were also tested for FM detection without interfering AM. The audiograms for the tested (right) ears of all 12 listeners are shown in Fig. 1. All had audiometric thresholds $\leq 60 \mathrm{~dB}$ hearing level (HL) between 0.25 and $4 \mathrm{kHz}$. The severity of hearing loss was classified as mild to moderate based on audiometric thresholds. The audiometric thresholds at the tested frequency $(0.5 \mathrm{kHz})$ ranged between 30 and $50 \mathrm{~dB} \mathrm{HL}$ $($ mean $=40 \mathrm{~dB} \mathrm{HL} ; \mathrm{SD}=5.6 \mathrm{~dB})$. For the 9 HIo listeners who were tested for FM detection without interfering AM, the audiometric threshold at the tested frequency ranged

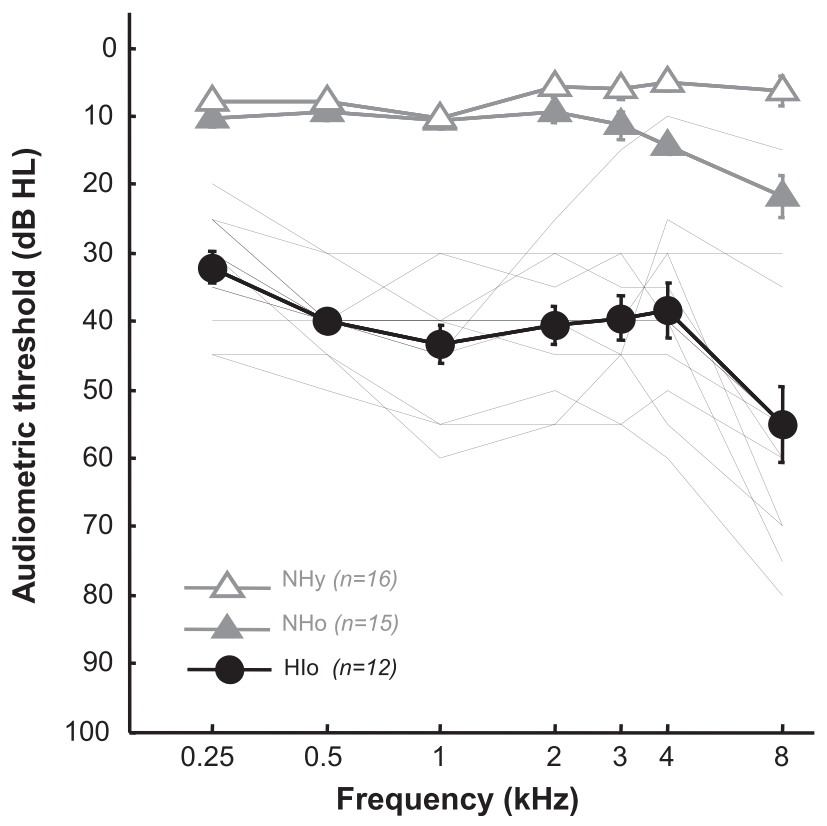

FIG. 1. Individual (thin lines) and mean (filled circles and black thick lines) audiometric thresholds for the older hearing-impaired (HIo) listeners for the right (tested) ear. Mean thresholds for the young normal-hearing (NHy) group are shown by open triangles and mean thresholds for the older normal-hearing (NHo) group are shown by filled gray triangles. Error bars indicate \pm 1 standard error of the mean. They are omitted when they would be smaller than the size of the symbol used to plot the data. between 30 and $45 \mathrm{~dB}$ HL $($ mean $=38 \mathrm{~dB} \mathrm{HL} ; \mathrm{SD}=5 \mathrm{~dB})$. The cochlear origin of hearing loss was diagnosed based on air-conduction, bone-conduction and impedance audiometry. Eleven out of the 12 HIo listeners participated in a previous study that investigated temporal integration for AM detection (Wallaert et al., 2017).

\section{Normal-hearing listeners}

Sixteen NHy listeners aged between 22 and 28 years (mean $=24$ years; $S D=2$ years) and 15 NHo listeners aged between 47 and 66 years (mean $=55$ years; $S D=8$ years) were tested for FM detection with interfering AM and for AM detection. The data for 15 of the NHy listeners and 14 of the NHo listeners were reported by Wallaert et al. (2016) and Wallaert et al. (2017). All of these NHy and NHo listeners had audiometric thresholds less than $20 \mathrm{~dB}$ HL for the tested (right) ear for octave frequencies between 0.25 and $3 \mathrm{kHz}$, but some of the NHo listeners had elevated audiometric thresholds for frequencies above $3 \mathrm{kHz}$. The mean audiometric threshold at $0.5 \mathrm{kHz}$ was $7.8 \mathrm{~dB} \mathrm{HL}(\mathrm{SD}=3.1 \mathrm{~dB})$ for the NHy group and $9.3 \mathrm{~dB} \mathrm{HL}(\mathrm{SD}=5 \mathrm{~dB})$ for the NHo group. Audiometric thresholds at $0.5 \mathrm{kHz}$ were not significantly different between the NHy and NHo groups [t(29) $=-1.03 ; p=0.313]$. A second $t$-test showed no significant difference in age between the NHo and HIo groups [t(25) $=-1.11 ; p=0.275]$.

Eleven of the listeners for each group were also tested for FM detection without interfering AM. For the $11 \mathrm{NHy}$ and 11 NHo listeners who were tested for FM detection without interfering $\mathrm{AM}$, the mean audiometric threshold at $0.5 \mathrm{kHz}$ was $7.7 \mathrm{~dB} \mathrm{HL}(\mathrm{SD}=3.4 \mathrm{~dB})$ for the NHy group and $9.5 \mathrm{~dB} H L(\mathrm{SD}=5.7 \mathrm{~dB})$ for the NHo group. A $t$-test showed no significant difference in audiometric thresholds at $0.5 \mathrm{kHz}$ between the two NH groups $[t(20)=0.91 ; p=0.375]$. A second $t$-test showed no significant difference in age between the NHo and HIo groups $[t(21)=1.31 ; p=0.204]$. The data for 8 of the 11 NHy listeners were reported by Wallaert et al. (2016).

All listeners were fully informed about the goal of the study and provided written consent before their participation. This study was approved by the local ethical committee of University Paris Descartes (CERES, No. IRB: 20143200001072 and No. IRB: 20153600001072).

\section{B. Procedure}

FMDTs were measured using a two-interval forcedchoice adaptive procedure estimating the $70.7 \%$ point on the psychometric function (Levitt, 1971). Each trial contained a target (with FM) and a standard (without FM) stimulus, in random order, with a silent interval of $600 \mathrm{~ms}$ between them. Listeners were asked to indicate which interval contained the "extra" pitch fluctuation. The root-mean-square level of the two stimuli was, on average, the same. To reduce the ability to use possible cues relating to small overall loudness differences, the levels of the standard and target stimuli were roved independently over a range of $\pm 1.5 \mathrm{~dB}$. The onset and offset ramps had durations of 250 and $25 \mathrm{~ms}$ for the modulation rates of 2 and $20 \mathrm{~Hz}$, respectively (the ramp duration 
was equal to half the period of the modulation). In conditions with interfering AM, the AM was present in both intervals of a trial, and was therefore uninformative. For both the AM and FM detection tasks, the duration of each signal was determined by the number of modulation cycles, $N$, and the modulation rate, $f_{m}$. For the FM detection task, $N$ was 2, 3, 4, 5 , or 9 cycles when FM was presented with interfering AM and 2, 3, or 4 cycles when FM was presented without any interfering AM. For the AM detection task, $N$ was 2, 3, 4, 5, or 9 cycles. Each interval was marked by a light on the computer screen. Visual feedback as to the correct response was provided after each response.

The tracking variable was the frequency excursion in $\mathrm{Hz}, \Delta f$ (see Sec. II C for details). A run started with the tracking variable well above the estimated detection threshold for all groups (the starting value of $\Delta f$ was $20 f_{m}$ for both rates). Following two consecutive correct responses, the value of $\Delta f$ was decreased, while following one incorrect response it was increased. The value of $\Delta f$ was changed by a factor of $1.5625\left(1.252^{2}\right)$ until one reversal had occurred, and then by a factor of 1.25. Fourteen reversals were obtained for each run, and the threshold estimate for that run was taken as the geometric mean of the values of $\Delta f$ at the last six reversals. Two threshold estimates were obtained for each condition. The final estimate of the threshold was taken as the geometric mean of the two threshold estimates.

\section{Stimuli}

The stimuli were identical to those used by Wallaert et al. (2016). All stimuli were generated digitally at a sampling rate of $44.1 \mathrm{kHz}$. They were presented using Sennheiser HD 280 pro headphones (Old Lyme, CT) and an external soundcard (ECHO Indigo DJ 1-2, Santa Barbara, California; 16-bit resolution) at a mean SL of $40 \mathrm{~dB}$, except for 3 HIo listeners with absolute thresholds of $40 \mathrm{~dB} \mathrm{HL}$ or more, for whom the level was set to $80 \mathrm{~dB}$ SPL. Levels were calibrated using a Bruel \& Kjaer 2250 sound level meter and ear simulator type 4153 (Naerum, Denmark) complying with IEC 60318-1. Stimuli were presented only to the right ear.

For the FMDTs measured with interfering AM, the standard stimulus contained AM but not FM. The target stimulus contained both AM and FM. Equation (1) describes the target stimulus $\mathrm{T}(t)$,

$$
\mathrm{T}(t)=\mathrm{AM}(t) \sin \left[\left(2 \pi f_{c} t+\Phi\right)+\beta\left(\sin \left(2 \pi f_{m} t+\varphi\right)\right)\right],
$$

with $\operatorname{AM}(t)=\left[1+m \sin \left(2 \pi f_{m} t+3 \pi / 2\right)\right]$ with $m=0.33$ and $\beta=\Delta f / f_{m}$, where $\Delta f$ is the frequency excursion in $\mathrm{Hz}, f_{m}$ is the modulation rate $(2$ or $20 \mathrm{~Hz}), \varphi$ is the starting phase of the FM, $f_{c}$ is the carrier frequency $(500 \mathrm{~Hz}), \Phi$ is the starting phase of the carrier, and $t$ is time in s. The values of $\varphi$ and $\Phi$ were randomly and independently chosen for each stimulus. The interfering AM had a fixed modulation index of 0.333 , corresponding to a peak-to-valley ratio of $6 \mathrm{~dB}$, as in Moore and Glasberg (1989). It was chosen to be large enough to disrupt cues for FM detection based on changes in excitation level, but not so large that it would induce substantial levelrelated pitch shifts (Verschuure and van Meeteren, 1975;
Emmerich et al., 1989). Although the starting phase of the AM was fixed, its phase relative to that of the FM was random (because $\varphi$ was chosen randomly), so the AM should still have been effective in disrupting the use of temporalenvelope cues. For the standard stimulus, $\Delta f$ was set to 0 . For the FMDTs measured without interfering AM, the value of $m$ was set to 0 .

\section{RESULTS}

\section{A. AMDTs versus FMDTs with and without AM ( $N=2-4$ cycles)}

Figure 2 shows mean AMDTs [top panels, taken from Wallaert et al. (2016) and Wallaert et al. (2017)] and FMDTs (bottom panels) as a function of $N$, for the NHy (left-most panels), NHo (middle panels), and HIo (rightmost panels) listeners. FMDTs (lower panels) were measured with (grey lines) and without (black lines) interfering AM. In each panel, the filled circles and open triangles show the thresholds for $f_{m}=2 \mathrm{~Hz}$ and $f_{m}=20 \mathrm{~Hz}$, respectively. AMDTs are expressed as $20 \log _{10} m(\mathrm{~dB})$ and FMDTs are expressed as $\Delta f(\mathrm{~Hz}, \log$ scale $)$.

The reader is referred to Wallaert et al. (2016) for analysis of the AMDTs and FMDTs for the NHy and NHo listeners and for discussion of the effects of age on AM and FM detection. The FMDTs for the HIo listeners are comparable to those reported previously (Moore and Skrodzka, 2002; Lacher-Fougère and Demany, 1998; Buss et al., 2004; Strelcyk and Dau, 2009; Ernst and Moore, 2012; Johannesen et al., 2016; Paraouty et al., 2016) for FM rates at or below $20 \mathrm{~Hz}$ and carrier frequencies below $1 \mathrm{kHz}$. In addition, the results are consistent with those of Kortlang et al. (2016), who assessed the detection of random slow FM $(1-4 \mathrm{~Hz})$, with and without superimposed AM.

A mixed-model analysis of variance (ANOVA) was conducted on the AMDTs with between-subjects factor
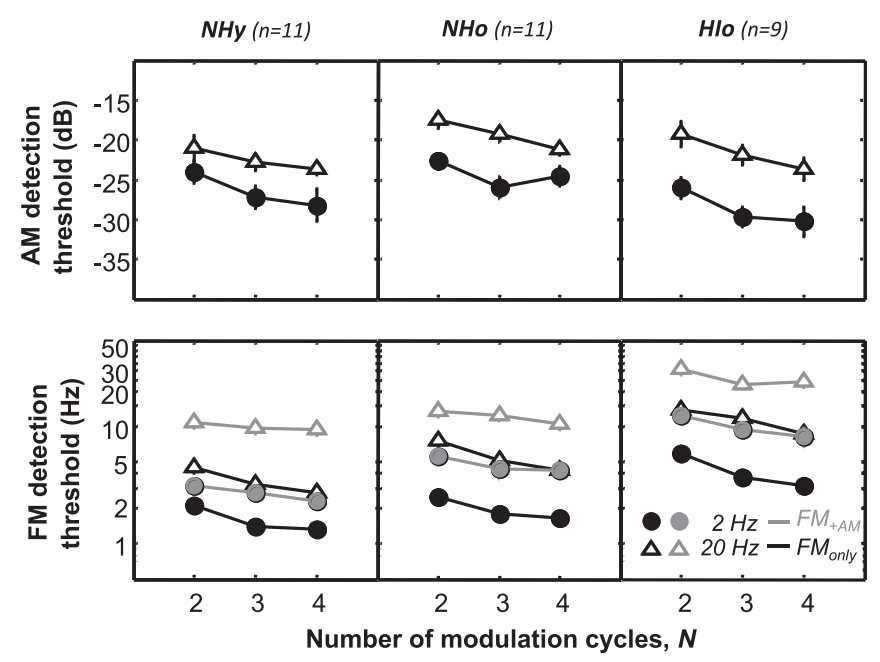

FIG. 2. Mean AMDTs (top panels) and FMDTs (bottom panels) as a function of $\mathrm{N}$ for the $\mathrm{NHy}$ (left panels), NHo (middle panels), and HIo (right panels) groups. In each panel, the filled circles and open triangles show the thresholds for $f_{m}=2$ and $20 \mathrm{~Hz}$, respectively. FMDTs measured without interfering AM ("FM-only" condition) are shown as black symbols and black lines. FMDTs measured with interfering AM ("FM+AM" condition) are shown as gray symbols and gray lines. 
group (3 levels: $\mathrm{NHy}, \mathrm{NHo}$, and HIo) and within-subjects factors $N$ (3 levels) and $f_{m}$ (2 levels). The effect of $f_{m}$ was significant $[F(1,28)=47.53, p<0.001]$. For all three groups, AMDTs were lower (i.e., better) for $f_{m}=2 \mathrm{~Hz}$ than for $f_{m}=20 \mathrm{~Hz}$. The effect of $N$ was significant $[F(2,56)=26.01$, $p<0.001]$. For each $f_{m}$ and each group, AMDTs decreased (i.e., improved) with increasing $N$. The interaction between $N$ and $f_{m}$ was not significant $[F(2,56)=1.43, p=0.24]$, indicating that AMDTs decreased similarly with increasing $N$ for the two modulation rates. The interaction between $N$ and group was not significant $[F(4,56)<1, p=0.80]$. This indicates that the temporal integration of AM cues was similar for all groups. This appears to be inconsistent with the results of Wallaert et al. (2017), who found greater temporal integration of $\mathrm{AM}$ for HIo listeners than for $\mathrm{NHy}$ and $\mathrm{NHo}$ listeners. However, the study of Wallaert et al. (2017) included higher values of $N$ (5 and 9), making it easier to detect differences across groups. The effect of group was significant $[F(2,28)$ $=3.46, p=0.045]$. Post hoc comparisons were based on Fisher's protected least-significant difference test. For all conditions, AMDTs were higher for the NHo than for the NHy group $(p=0.049)$, and were lower for the HIo group than for the NHo group $(p=0.022)$, indicating a deleterious effect of greater age and a beneficial effect of hearing loss. The interaction between $f_{m}$ and group was not significant $[F(2,28)$ $=1.28, p=0.29$ ], indicating that the effect of $f_{m}$ was similar for the three groups. None of the other interactions involving group were significant $\left[N \times f_{m} \times\right.$ group: $\left.F(4,56)<1\right]$.

A mixed-model ANOVA was conducted on the (logtransformed) FMDTs with within-subject factors presence/ absence of interfering $\mathrm{AM}$ (2 levels), $N$ ( 3 levels), and $f_{m}$ (2 levels), and between-subjects factor group ( 3 levels). The effect of group was significant $[F(2,28)=96.5 ; p<0.001]$, FMDTs being higher for the older groups than for the younger group, and being higher for the HIo group than for the NHo group, as shown by post hoc comparisons (both $p<0.001$ ). The effect of interfering AM was significant $[F(1,28)=350.4$; $p<0.001]$, FM detection being worse in the presence of interfering AM. The effect of $N$ was significant $[F(2,56)=94.3$; $p<0.001]$, FMDTs decreasing with increasing $N$. The effect of $f_{m}$ was significant $[F(1,28)=308.9 ; p<0.001]$, FM detection being better for $f_{m}=2 \mathrm{~Hz}$ than for $f_{m}=20 \mathrm{~Hz}$. The interaction between interfering $\mathrm{AM}$ and $f_{m}$ was significant $[F(1,28)=5.8 ; p=0.023]$, the detrimental effect of the interfering AM being greater for $f_{m}=20 \mathrm{~Hz}$ than for $f_{m}=2 \mathrm{~Hz}$ $(p<0.001)$. This finding is consistent with previous work (Moore and Sek, 1996; Ernst and Moore, 2010, 2012) and with the notion that temporal-envelope cues play a greater role in FM detection at $f_{m}=20 \mathrm{~Hz}$ than at $f_{m}=2 \mathrm{~Hz}$. The interaction between interfering $\mathrm{AM}$ and $N$ was significant $[F(2,56)=5.6 ; p=0.006]$, the decrease in threshold with increasing $N$ being greater in the absence than in the presence of interfering AM for all groups of listeners and for both modulation rates. For example, for the NHo group the threshold for $f_{m}=20 \mathrm{~Hz}$ decreased from $7.5 \mathrm{~Hz}$ for $N=2$ to $4.2 \mathrm{~Hz}$ for $N=4$ for FM only (a factor of 0.56 ), while for FM $+\mathrm{AM}$, the threshold decreased from $13.7 \mathrm{~Hz}$ for $N=2$ to $10.7 \mathrm{~Hz}$ for $N=4$ (a factor of 0.78). The interaction between interfering $\mathrm{AM}$, group and $f_{m}$ was significant $[F(2,28)=5.8 ; p=0.008]$.
To analyze this interaction further, an additional mixedmodel ANOVA was conducted on the ratio of the FMDTs measured with and without interfering AM (giving a measure of the interference effect caused by the AM), with withinsubject factors $N$ (3 levels) and $f_{m}$ (2 levels), and betweensubjects factor group (3 levels). The interaction between group and $f_{m}$ was significant $[F(2,28)=4.895 ; p=0.015]$. Post hoc analysis indicated that the deleterious effect of interfering $\mathrm{AM}$ was greater at $f_{m}=20 \mathrm{~Hz}$ than at $f_{m}=2 \mathrm{~Hz}$ for the NHy group only $(p<0.001)$. This pattern of results is consistent with the idea that NHy listeners can detect FM for low carrier frequencies and very low FM rates using a cue other than that resulting from FM-to-AM conversion (Moore and Sek, 1994, 1996; Sek and Moore, 1995) and that the ability to use this cue is reduced with increasing age (He et al., 2007; Kortlang et al., 2016) and with hearing loss (Moore and Skrodzka, 2002; Paraouty et al., 2016).

\section{B. AMDTs compared to FMDTs with AM $(N=2-9$ cycles)}

Figure 3 shows mean AMDTs (top panels) and FMDTs measured with interfering AM (bottom panels) as a function of $N$ for the NHy (left-most panels), NHo (middle panels), and HIo (right-most panels) listeners.

A mixed-model ANOVA was conducted on the AMDTs with between-subjects factor group (3 levels: $\mathrm{NHy}, \mathrm{NHo}$, and HIo) and within-subjects factors $N$ (5 levels) and $f_{m}(2$ levels). The effect of $f_{m}$ was significant $[F(1,40)=73.79$, $p<0.001]$. For all groups, AMDTs were lower (i.e., better) for $f_{m}=2 \mathrm{~Hz}$ than for $f_{m}=20 \mathrm{~Hz}$. The effect of $N$ was significant $[F(4,160)=63.63, p<0.001]$. For each $f_{m}$ and each group, AMDTs decreased (improved) with increasing $N$. The interaction between $N$ and $f_{m}$ was significant $[F(4$, $160)=3.70, p=0.007]$. Consistent with the results of Wallaert et al. (2017), the interaction between $N$ and group was significant $[F(8,160)=3.68, p<0.001]$. The effect of group was significant $[F(2,40)=9.95, p<0.001]$. Post hoc comparisons showed that for all $N$, AMDTs were higher for the NHo group than for the NHy group $(p=0.002)$, but were
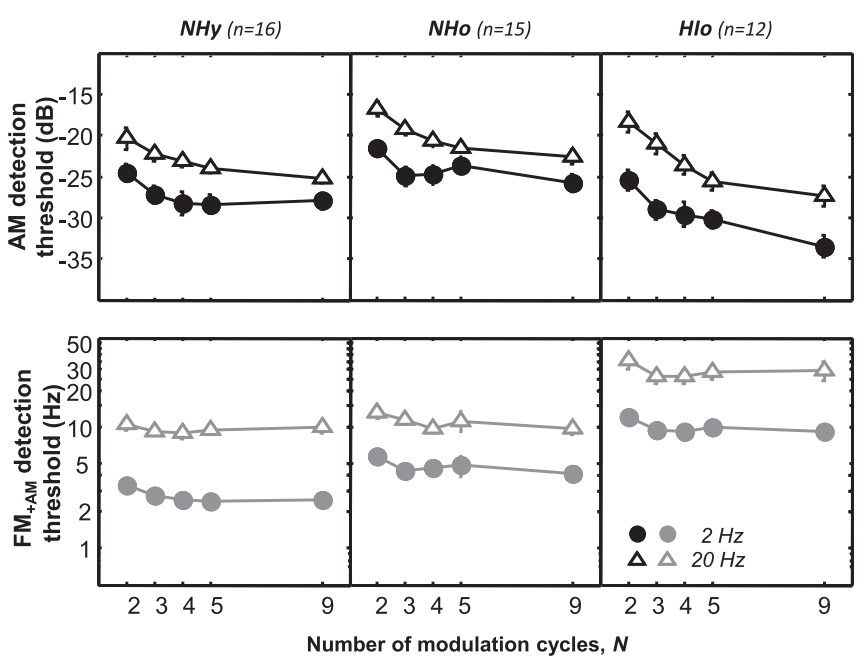

FIG. 3. As Fig. 2, except for the greater range of $\mathrm{N}$, and the fact that FMDTs were measured only with interfering AM. 
lower for the HIo group than for the NHo group $(p<0.001)$, indicating a deleterious effect of greater age but a beneficial effect of hearing loss. The interaction between $f_{m}$ and group $[F(2,40)=1.65, p=0.20]$ was not significant: the effect of $f_{m}$ was similar for the three groups. None of the other interactions involving group was significant $\left(N \times f_{m} \times\right.$ group: $[F(8,160)<1])$.

A linear regression analysis was conducted on the individual AMDTs [log-transformed AMDTs versus $\log (\mathrm{N})$ ]. A repeated-measures ANOVA was conducted on the resulting slopes with between-subject factor group (3 levels) and within-subjects factor $f_{m}$ (2 levels). There was a significant effect of $f_{m}[F(1,40)=7.64, p=0.008]$, a significant effect of group $[\mathrm{F}(2,40)=9.1, p<0.001]$ and no significant interaction $[F(2,40)<1, p=0.611]$. Post hoc analyses showed that temporal integration for AM detection was: (1) greater at $20 \mathrm{~Hz}$ than at $2 \mathrm{~Hz}$; (2) greater for the HIo than for the two $\mathrm{NH}$ groups (both $p<0.002$ ).

A mixed-model ANOVA was conducted on the FMDTs with between-subjects factor group (3 levels) and withinsubjects factors $N$ (5 levels) and $f_{m}$ (2 levels). The effect of $f_{m}$ was significant $[F(1,40)=314.6, p<0.001]$. For all groups, FMDTs were lower for $f_{m}=2 \mathrm{~Hz}$ than for $f_{m}$ $=20 \mathrm{~Hz}$. The effect of $N$ was significant $[F(4,160)=14.5$, $p<0.001]$. The interaction between $N$ and $f_{m}$ was not significant $[F(4,160)<1]$, suggesting that FMDTs decreased similarly with increasing $N$ for the two modulation rates. The interaction between $N$ and group was not significant $[F(8$, $160)<1$ ], indicating that FMDTs decreased similarly with increasing $N$ for all groups. The effect of group was significant $[F(2,40)=66.2, p<0.001]$. For all conditions, FMDTs were higher for the NHo than for the NHy group $(p=0.004)$ and were higher for the HIo than for the NHo group $(p<0.001)$, indicating deleterious effects of both age and hearing loss. There was a significant interaction between $f_{m}$ and group $[F(2,40)=3.6, p=0.03]$. The ratio between FMDTs for $f_{m}=20$ and $2 \mathrm{~Hz}$ was 3.6 for the NHy group, 2.4 for the NHo group, and 2.9 for the HIo group. The difference between the NHy and NHo groups is consistent with the finding of Wallaert et al. (2016) that age had a greater effect on FMDTs at $2 \mathrm{~Hz}(p<0.001)$ than at $20 \mathrm{~Hz}(p=1)$. This might reflect the effects of age on the use of TFS cues for $2-\mathrm{Hz}$ FM detection. Hearing loss was associated with an increase in FMDTs for both modulation rates (all $p<0.001$ ), consistent with the results of Lacher-Fougère and Demany (1998), Moore and Skrodzka (2002), and Strelcyk and Dau (2009). This may happen because hearing loss reduces the ability to use both temporal-envelope cues (reduced FMto-AM conversion because of broader auditory filters) and TFS cues. None of the other interactions involving group was significant.

\section{MODELING AM AND FM DETECTION}

\section{A. Rationale}

The present data combined with those reported by Wallaert et al. (2016) and Wallaert et al. (2017) show a differential effect of hearing loss on AM and FM detection and integration: FM detection was poorer for the HIo than for the
NHo group but temporal integration of FM cues was similar for the two groups, whereas AM detection was better for the HIo than for the NHo group and temporal integration of AM cues was greater for the HIo than for the NHo group. At first sight, this dissociation suggests different mechanisms for $\mathrm{AM}$ and FM detection and integration when $f_{c}$ is low (here, $500 \mathrm{~Hz}$ ) and suggests that hearing loss preserves the memory and decision processes responsible for temporal integration of FM cues. To assess whether this interpretation is correct, the FMDTs were compared with the predictions of an envelopeprocessing model whose parameters were initially adjusted to fit the AMDTs. The model is described in Sec. IV B.

\section{B. The model}

The general structure of the model is similar to that described by Wallaert et al. (2017). The first stage was a set of five linear gammatone filters (Patterson et al., 1995) that simulated the bandpass filtering of the basilar membrane in the cochlea. One gammatone filter (called the on-frequency filter) was centered on the carrier frequency of the signal $(500 \mathrm{~Hz})$. The remaining four filters (the off-frequency filters) were centered one and two Cams (units of the $\mathrm{ERB}_{\mathrm{N}^{-}}$ number scale; Glasberg and Moore, 1990) above and below the carrier frequency. In what follows, the sequence of stages based on the output of each of the simulated auditory filters is referred to as a channel. In the second stage, the outputs of the five gammatone filters were half-wave rectified. For simulating normal hearing, the rectified output of the onfrequency filter was processed by a broken-stick input-output function, which applied compression for levels above a given threshold level, chosen so that compression occurred only for stimuli with levels above $40 \mathrm{~dB}$ SPL. The compression was implemented by raising the amplitude to the power 0.3 . The rectified signals at the outputs of the four off-frequency filters were not compressed. This crudely simulates the physiological finding that compression in the cochlea occurs mainly for input frequencies close to the characteristic frequency of the place whose response is being measured (Robles and Ruggero, 2001).

The signals derived from the outputs of the five auditory filters were highpass filtered (cutoff $=2.5 \mathrm{~Hz}$; rolloff $=20 \mathrm{~dB} /$ decade) to simulate the effects of adaptation in the AM domain (Tchorz and Kollmeier, 1999). The resulting signals were passed through a set of 10 bandpass (modulation) filters, one set for each channel, to simulate frequencyselective processing in the AM domain (Dau et al., 1997a,b). The modulation filters had a $Q$ value of 1 and a rolloff of $\pm 20 \mathrm{~dB} /$ decade (Ewert and Dau, 2000; Ewert et al., 2002; Lorenzi et al., 2001; Sek and Moore, 2002). The center frequencies of the modulation bandpass filters ranged between 2 and $120 \mathrm{~Hz}$ (Moore et al., 2009) and were spaced logarithmically. For each modulation filter centered below $10 \mathrm{~Hz}$, the waveform at the output of the filter was passed on for further processing, while for each filter centered at and above $10 \mathrm{~Hz}$ only the Hilbert envelope of the output was passed on. This was done to simulate the loss of sensitivity to envelope phase for rates above $10 \mathrm{~Hz}$ (Dau et al., 1997a,b). Each envelope of the envelope (the so-called "venelope" Ewert et al., 2002) 
was scaled so that the root-mean-square value at the output of modulation filters was the same before and after the Hilbert transformation. Figure 4 shows the final model outputs for each gammatone filter and each modulation filter in response to an FM tone $\left[f_{\mathrm{c}}=500 \mathrm{~Hz} ; N=9 ; f_{m}=2 \mathrm{~Hz}\right.$ (left panels) and $f_{m}=20 \mathrm{~Hz}$ (right panels)] with a suprathreshold frequency excursion $\left(\Delta f=20 f_{m}\right)$. The figure shows the final output patterns for the on-frequency and off-frequency channels. For $f_{m}=2 \mathrm{~Hz}$, the fluctuation rate of the output was twice $f_{m}$ for the on-frequency channel, and there was a large phase shift in output between the off-frequency channels centered above and below $500 \mathrm{~Hz}$. These features were not clearly apparent for $f_{m}=20 \mathrm{~Hz}$, because of venelope extraction.

The outputs of the modulation filters (the envelope for $f_{m}<10 \mathrm{~Hz}$; the venelope for $f_{m} \geq 10 \mathrm{~Hz}$ ) were down-sampled by a factor 10 and then "degraded" by two sources of internal noise, which were introduced to limit the performance of the model. These noises were: (1) an additive noise, $\varepsilon_{a d d}$, and (2) an additive time-varying memory noise, characterized by parameter $\varepsilon_{\text {mem }}$. Both noises were modeled as Gaussian noises with zero mean and with magnitudes specified by their SD $\sigma$. Randomly drawn samples of each noise were added independently to each sample point of the outputs of the modulation filters. $\varepsilon_{\text {add }}$ was introduced to limit intensity discrimination and AM sensitivity (Dau et al., 1997 a,b). $\varepsilon_{m e m}$ was introduced to simulate imperfect retention of temporal-envelope information (Ardoint et al., 2008; Wallaert et al., 2017). This noise primarily played a role for

$$
\mathrm{fm}=2 \mathrm{~Hz}
$$
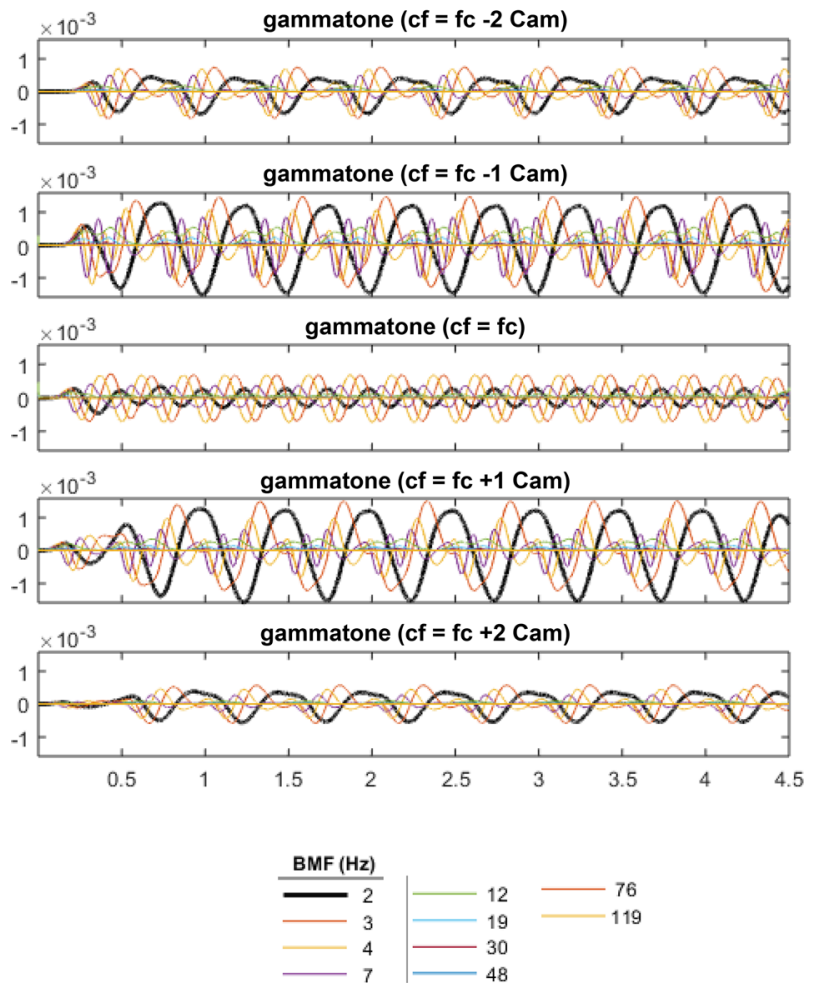

very long stimuli (e.g., for 4.5-s stimuli, as used when $N=9$ and $f_{m}=2 \mathrm{~Hz}$ ). The SD of the memory noise increased backward in time according to an exponential function with a 1.4-s half life. The parameter $\sigma_{m e m}$ corresponds to the SD of the memory noise. The resulting (noisy) signals were used as input to the decision stage of the model. Equation (2) describes the noise terms and how they were combined with model signals,

$$
I_{\text {noisy }}(t, c f, B M F)=I(t, c f, B M F)+\varepsilon_{a d d}+\varepsilon_{m e m} e^{t / \tau},
$$

with $\varepsilon_{a d d}=N\left(0, \sigma_{a d d}\right)$ and $\varepsilon_{m e m}=N\left(0, \sigma_{m e m}\right)$.

$N$ here is meant to denote a sample from the normal distribution, $t$ is the time sample index in memory, increasing in the backward direction, i.e., the time relative to the end of the stimulus ( $t=0$ corresponds to the most recent sample), $c f$ is the center frequency of the gammatone filter, and $B M F$ is the center frequency of the modulation filter (its "best modulation frequency"). The internal representation, $I(\cdots)$ is a 50 -dimensional vector $(5$ channels $\times 10$ modulation filters per channel), and the noise samples were independent for each dimension and each time sample.

The decision stage was realized as a simplified version of the optimal detector described by Dau et al. (1997a,b). The output of the model was computed for the two stimuli in each trial. A template was generated at the beginning of each simulated threshold measurement (that is, for the first trial of each adaptive staircase) as the difference between the model output in response to the target and standard stimuli. This was done with the two internal noise sources set to zero.
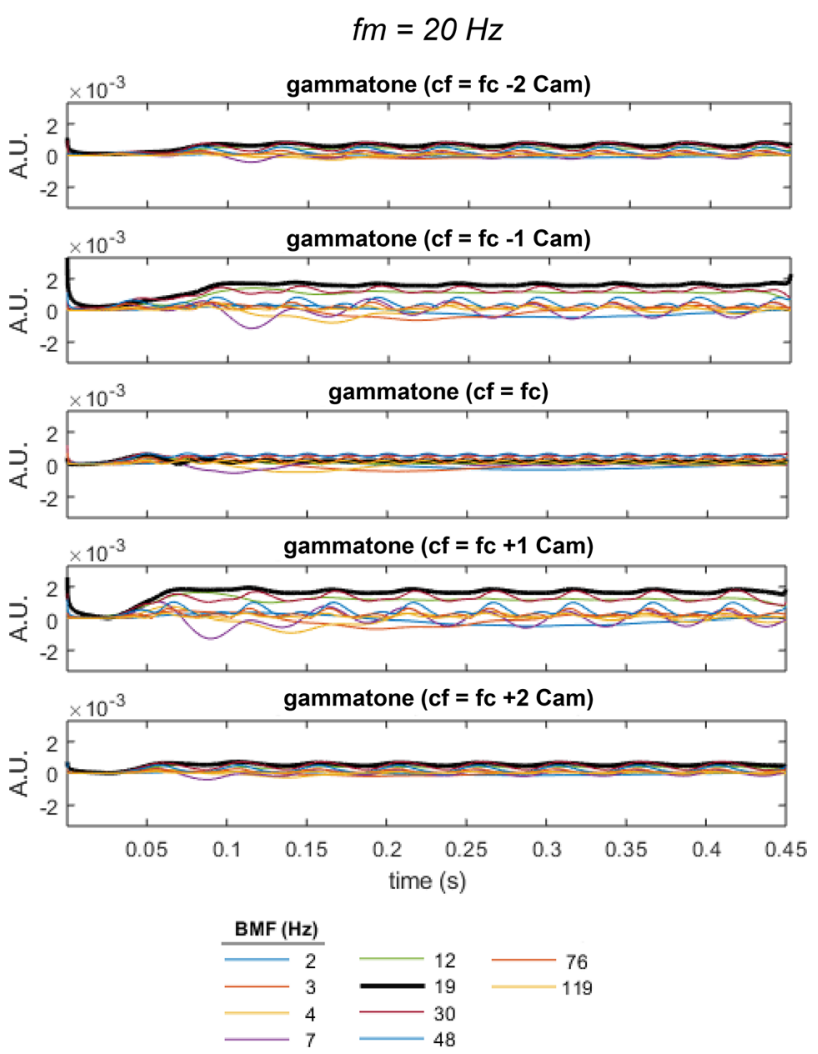

FIG. 4. (Color online) Internal representation of a $2-\mathrm{Hz}$ (left panels) or $20-\mathrm{Hz}$ (right panels) FM signal (500-Hz carrier) for each channel [with center frequency (cf)] and each modulation filter [with best modulation frequency (BMF)]. The black line indicates the output of the modulation filter closest to the target rate $(2$ or $19 \mathrm{~Hz})$. 
Note that this largely cancelled out the effects of the added $\mathrm{AM}$ on the internal representation, and therefore it represented an idealized version of what listeners do. For each subsequent trial, the template was cross-correlated with the model output for each interval in that trial. The lags used by the cross-correlation device were restricted to \pm 1 modulation cycle. The normalized cross-correlation was computed separately for each channel and each modulation filter. The correlation functions were then summed across all channels to estimate the lag corresponding to the best match. The interval leading to the largest cross-correlation was taken as the target interval identified by the model for that trial. Stimuli were generated as for the behavioral experiment including the roving of overall level. Ninety thresholds were simulated and averaged for each condition.

\section{Choice of model parameters}

To model the data for the NHy and NHo groups, the bandwidths of the gammatone filters were set to either 0.7 or $1 \mathrm{ERB}_{\mathrm{N}}$ (Glasberg and Moore, 1990). The same values were used for these two groups because auditory filters do not broaden with increasing age when the audiometric threshold remains normal (Lutman et al., 1991; Peters and Moore, 1992). The values of $\mathrm{ERB}_{\mathrm{N}}$ represent average values of the bandwidths of the auditory filters estimated using data from simultaneous masking experiments (mostly with notched noise). The smaller value of $0.7 \mathrm{ERB}_{\mathrm{N}}$ was used based on the suggestion that human cochlear tuning may be sharper than estimated using simultaneous notched-noise masking (Oxenham and Shera, 2003) and also based on previous attempts to model AM and FM detection for NH listeners, which showed that filters with bandwidths less than $1 \mathrm{ERB}_{\mathrm{N}}$ were required to give a good fit to the data (Moore and Sek, 1994). To model the data for the HIo group, the bandwidths of the gammatone filters were set to either 1.4 or $2 \mathrm{ERB}_{\mathrm{N}}$, simulating the reduced frequency selectivity associated with moderate sensorineural hearing loss (Glasberg and Moore, 1986; Moore, 2007). The compression parameter was set to 0.3 to model the data for the NHy and NHo groups, simulating "normal" cochlear compression for the on-frequency filter. For the HI group, the compression parameter was set to 1.0 , simulating the loss of compression associated with cochlear hearing loss. The cutoff frequency of the highpass filter used to model adaptation was chosen so as to predict the relative values of the AMDTs for the two modulation rates. The values of the two noise parameters were chosen by first adjusting parameter $\varepsilon_{\text {add }}$ and then parameter $\varepsilon_{m e m}$ so as to fit the AMDTs for the NHy listeners. Subsequently, parameter $\varepsilon_{\text {add }}$ was adjusted to fit the AMDTs for the NHo and HIo listeners, while keeping $\varepsilon_{\text {mem }}$ constant. Consistent with the modeling described by Wallaert et al. (2017), the values of $\varepsilon_{\text {add }}$ obtained in this way were higher than for the NHy listeners by a factor 1.3 for the NHo listeners and by a factor 5.3 for the HIo listeners.

\section{Modeling results}

Figures 5-8 show the predictions of the model as symbols connected by dashed lines for AM detection (top
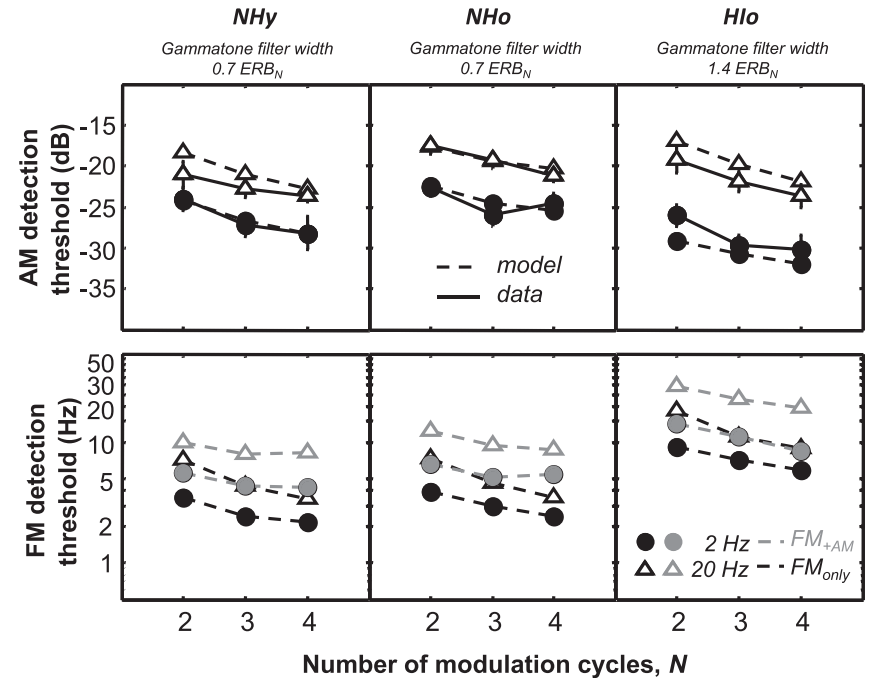

FIG. 5. Predicted AMDTs and FMDTs (dashed lines) for the models simulating the $\mathrm{NHy}, \mathrm{NHo}$, and HIo listeners. The widths of the gammatone filters were set to $0.7 \mathrm{ERB}_{\mathrm{N}}$ for the $\mathrm{NHy}$ and $\mathrm{NHo}$ models, and $1.4 \mathrm{ERB}_{\mathrm{N}}$ for the HIo model. Obtained AMDTs are shown by black symbols and continuous black lines in the top panels. Otherwise as Fig. 2.

panels) and FM detection (bottom panels). Black lines and symbols denote AMDTs and FMDTs measured without interfering AM. Gray lines and symbols denote FMDTs measured with interfering AM. Symbols connected by solid lines (when present) show the obtained thresholds.

\section{Effect of simulated auditory filter width on predicted AMDTs}

Consider first the effect of the bandwidths of the simulated auditory filters for the two $\mathrm{NH}$ groups on $\mathrm{AM}$ detection (top-left and top-middle panels in Figs. 5-8). Generally, the data were fitted more accurately with the bandwidth of 0.7 $\mathrm{ERB}_{\mathrm{N}}$ than with the bandwidth of $1 \mathrm{ERB}_{\mathrm{N}}$, especially for the $20-\mathrm{Hz}$ rate. This is consistent with the proposal that the cochlear filters in NH ears are somewhat sharper than estimated using simultaneous notched-noise masking (Oxenham
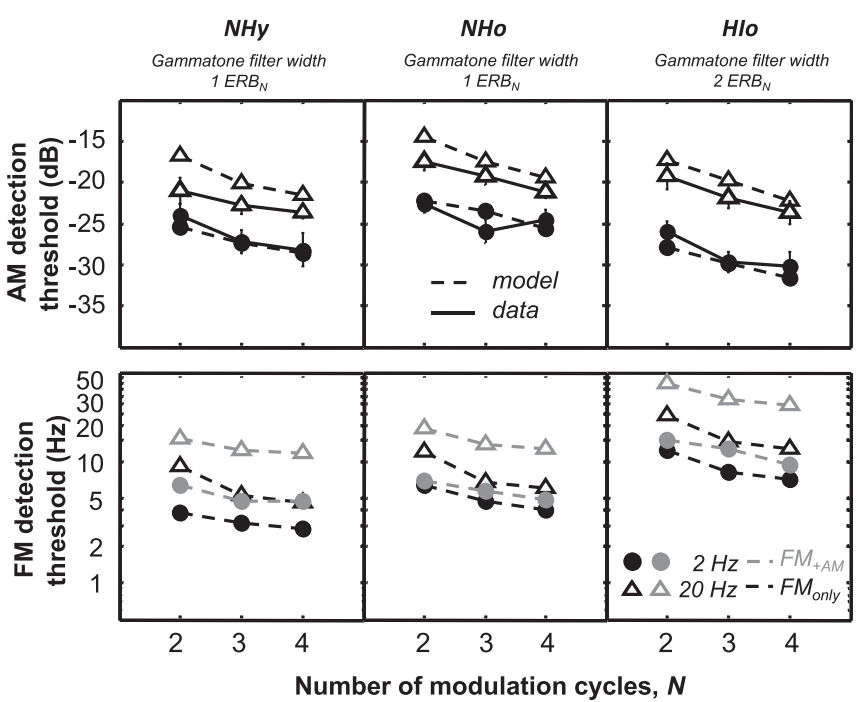

FIG. 6. As Fig. 5, but with the widths of the gammatone filters set to 1 $\mathrm{ERB}_{\mathrm{N}}$ for the NHy and NHo models, and $2 \mathrm{ERB}_{\mathrm{N}}$ for the HIo model. 


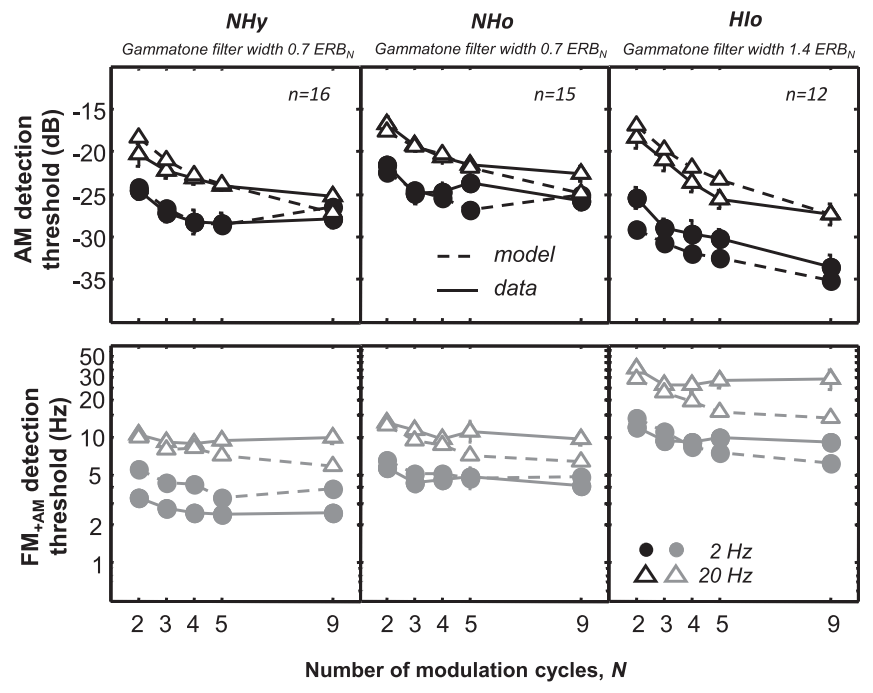

FIG. 7. Obtained (solid lines) and predicted (dashed lines) AMDTs (top) and FMDTs (bottom) for each group. The widths of the gammatone filters were set to $0.7 \mathrm{ERB}_{\mathrm{N}}$ for the $\mathrm{NHy}$ and $\mathrm{NHo}$ models, and $1.4 \mathrm{ERB}_{\mathrm{N}}$ for the HIo model. FMDTs were measured only with interfering AM.

and Shera, 2003). The AM-detection data for the HIo listeners were fitted roughly equally well using the simulated auditory filters with bandwidths of 1.4 and $2 \mathrm{ERB}_{\mathrm{N}}$.

\section{Effects of age and hearing loss on predicted AMDTs}

The model predictions shown in the top panels of Figs. 5-8 captured the effects of AM rate, $N$ and group (i.e., the effects of age and hearing loss) on the AMDTs reasonably well. However, the model made slightly less accurate predictions than the model described by Wallaert et al. (2017). For example, the model predicted greater temporal integration for AM than observed for the NHy listeners when $f_{m}=20 \mathrm{~Hz}$ (see the top-left panels in Figs. 7 and 8), and lower AM thresholds than observed for the HIo listeners when $f_{m}=2 \mathrm{~Hz}$ (top-right panels of Figs. 5-8). However, the discrepancy in the latter case was small when the widths of the simulated filters were $2 \mathrm{ERB}_{\mathrm{N}}$ (see Figs. 6 and 8) and the
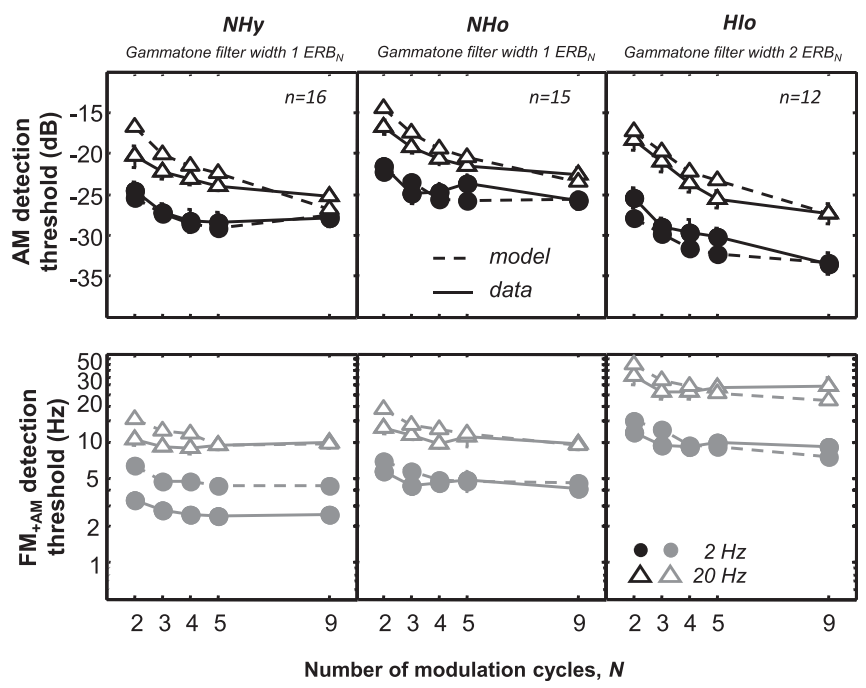

FIG. 8. As Fig. 7, but with the widths of the gammatone filters set to 1 $\mathrm{ERB}_{\mathrm{N}}$ for the NHy and NHo models, and $2 \mathrm{ERB}_{\mathrm{N}}$ for the HIo model. average absolute deviations between the obtained and predicted thresholds (across $N$ ) were reasonably small, ranging from 0.2 to $2 \mathrm{~dB}$ across conditions and groups.

\section{Effects of age and hearing loss on predicted FMDTs}

The model predictions shown in the bottom panels of Figs. 5-8 captured some of the main trends in the data for FM detection. Specifically, for both values of the widths of the gammatone filters, the predictions captured the effects of modulation rate (the predicted FMDTs were always lower for $f_{m}=2$ than for $f_{m}=20 \mathrm{~Hz}$ ), the effects of $N$ (the predicted FMDTs improved gradually with increasing $N$ ), and the effects of interfering AM (the predicted FMDTs were always poorer in the presence of interfering AM). Consistent with the general form of the results, the model predicted that FMDTs would be higher with greater age and with hearing loss. However, the predicted FMDTs were often higher than the obtained FMDTs, especially for the NHy group in the presence of interfering $\mathrm{AM}$ and for $f_{m}=2 \mathrm{~Hz}$ (see, for example, the bottom-left panels in Figs. 7 and 8). The discrepancy between the predictions and the data was somewhat less when the width of the simulated gammatone filters was 0.7 $\mathrm{ERB}_{\mathrm{N}}$ than when it was $1 \mathrm{ERB}_{\mathrm{N}}$, (compare Figs. 7 and 8), but it nevertheless remained quite large for the $0.7 \mathrm{ERB}_{\mathrm{N}}$ bandwidth.

As can be seen by comparing Figs. 2 and 5 and 6 , FMDTs without and with interfering AM with $N=2,3$, and 4 were predicted more accurately when the widths of the gammatone filters were set to $0.7 \mathrm{ERB}_{\mathrm{N}}$ for both $\mathrm{NH}$ model and $1.4 \mathrm{ERB}_{\mathrm{N}}$ for the HIo model. The goodness of fit was assessed by taking the absolute value of the logarithm of the ratio between predicted and obtained FMDTs and averaging this across values of $N$ and the presence/absence of AM; the smaller this average, called log-AV, the better is the fit. For the $\mathrm{NH}$ model, log-AV was 0.12 when the filter width was $0.7 \mathrm{ERB}_{\mathrm{N}}$ and 0.21 when it was $1 \mathrm{ERB}_{\mathrm{N}}$. For the HIo model, $\log$-AV was 0.12 when the filter width was $1.4 \mathrm{ERB}_{\mathrm{N}}$ and 0.17 when it was $2 \mathrm{ERB}_{\mathrm{N}}$.

The use of sharper gammatone filters resulted in larger temporal-envelope fluctuations generated by FM-to-AM conversion and this allowed the model to predict lower FMDTs, that were more consistent with the data. However, for FMDTs obtained with interfering AM using a wider range of $N$ (Fig. 3), the predictions for the two older groups and $f_{m}=20 \mathrm{~Hz}$ were more accurate for the wider simulated auditory filters (Fig. 8) than for the narrower simulated auditory filters (Fig. 7).

The model predicted FMDTs with and without interfering $\mathrm{AM}$ (data in Fig. 2) more accurately for $f_{m}=20 \mathrm{~Hz}$ than for $f_{m}=2 \mathrm{~Hz}$. For the NHy model with filter width 0.7 $\mathrm{ERB}_{\mathrm{N}}, \log$-AV was 0.17 when $f_{m}=2 \mathrm{~Hz}$ and 0.07 when $f_{m}=20 \mathrm{~Hz}$. For the NHo model with filter width $0.7 \mathrm{ERB}_{\mathrm{N}}$, $\log$-AV was 0.23 when $f_{m}=2 \mathrm{~Hz}$ and 0.10 when $f_{m}=20 \mathrm{~Hz}$. For the HIo model with filter width $1.4 \mathrm{ERB}_{\mathrm{N}}, \log -\mathrm{AV}$ was 0.16 when $f_{m}=2 \mathrm{~Hz}$ and 0.07 when $f_{m}=20 \mathrm{~Hz}$. This is consistent with the idea that FMDTs for $f_{m}=20 \mathrm{~Hz}$ are based entirely on the use of temporal envelope cues, while some other cue or cues play a role for $f_{m}=2 \mathrm{~Hz}$. 


\section{Effect of interfering AM on predicted FMDTs}

Interestingly, the model did predict the greater detrimental effect of interfering AM on FMDTs for $f_{m}=20 \mathrm{~Hz}$ than for $f_{m}=2 \mathrm{~Hz}$. However, inconsistent with the data (Fig. 2 , bottom panels), the predicted effect of interfering $\mathrm{AM}$ was of similar magnitude for the $\mathrm{NHy}, \mathrm{NHo}$, and HIo models for both modulation rates (Figs. 5 and 6, bottom panels). For both values of the width of the gammatone filters, the ratio of predicted FMDTs with and without interfering AM (averaged across $N$ ) ranged between 1.3 and 1.6 for $f_{m}=2 \mathrm{~Hz}$ and was about 2 for $f_{m}=20 \mathrm{~Hz}$ for the NHy, NHo, and HIo models. Corresponding obtained ratios were 1.8 for the $\mathrm{NHy}$ listeners and 2.6 for the NHo and HIo listeners for $f_{m}=2 \mathrm{~Hz}$, and 2.8 for the NHy listeners, 2.1 for the NHo listeners, and 2.5 for the HIo listeners for $f_{m}=20 \mathrm{~Hz}$. This inconsistency between the data and predictions occurred because the predicted FMDTs obtained without interfering AM for $f_{m}=2 \mathrm{~Hz}$ were systematically higher (i.e., poorer) than the obtained FMDTs for all three groups. The predicted FMDTs obtained with interfering $\mathrm{AM}$ for $f_{m}=2 \mathrm{~Hz}$ were close to the obtained FMDTs. This pattern of results can be explained in one or both of two ways: (1) FM-to-AM cues contributed to FM detection at a low modulation rate $\left(f_{m}=2 \mathrm{~Hz}\right)$, but were insufficient to explain the experimental data; (2) the $\mathrm{NHy}$, $\mathrm{NHo}$, and HIo listeners used a cue distinct from temporalenvelope cues when detecting FM, and this extra cue (presumably, TFS) was still available — but to a lesser extentfor the NHo and HIo listeners. It appears that this additional cue can be used more effectively in the absence than in the presence of interfering AM.

\section{Temporal integration of FM}

Whatever the width of the gammatone filters, the predicted temporal integration for FM in the absence of interfering AM was roughly consistent with the data. However, temporal integration was measured using a narrow range of $N$ (2-4 cycles) in this condition. For both widths of the gammatone filters, the predicted temporal integration for FM in the presence of interfering $\mathrm{AM}$, when measured using a wide range of $N$ (2-9 cycles), was much higher than observed for each group of listeners for $f_{m}=20 \mathrm{~Hz}$. The discrepancy was smaller for $f_{m}=2 \mathrm{~Hz}$, but was still apparent for the HIo group. As shown in Fig. 7, when the assumed bandwidths of the auditory filters were small $\left(0.7 \mathrm{ERB}_{\mathrm{N}}\right.$ for the two $\mathrm{NH}$ groups and 1.4 $\mathrm{ERB}_{\mathrm{N}}$ for the HIo group), this "overintegration" effect led to predicted FMDTs that were below obtained FMDTs for large $N$. As shown in Fig. 8, when the assumed bandwidths of the auditory filters were larger $\left(1 \mathrm{ERB}_{\mathrm{N}}\right.$ for the two $\mathrm{NH}$ groups and $2 \mathrm{ERB}_{\mathrm{N}}$ for the HIo group), the predicted FMDTs were above the obtained FMDTs for small $N$.

\section{DISCUSSION}

\section{A. Effects of age and hearing loss on AM detection and temporal integration}

For each value of $N$ and for both AM rates, AMDTs for the HIo listeners were significantly lower (i.e., better) than those for the NHo listeners tested at the same SL. This beneficial effect of sensorineural hearing loss on AM sensitivity has been explained as reflecting the loss of cochlear compression and the consequent loudness recruitment for the $\mathrm{HI}$ listeners. The latter has the effect of increasing the strength of the perceived fluctuations in AM sounds (Moore et al., 1996) and thus, the sensitivity to AM, at least for low SLs (Jerger, 1962; Moore, 2007). The interaction between $N$ and group was significant, temporal integration of AM being greater for the HIo group than for the two NH groups. This effect was predicted by the model (see the top panels of Figs. 7 and 8), consistent with the idea that it arises from loss of amplitude compression in the cochlea. Overall the results are consistent with the notion that at least some aspects of "processing efficiency" related to the short-term storage of temporal-envelope information and decision making are spared by cochlear damage (Wallaert et al., 2017).

Overall, the model presented here made slightly less accurate predictions of AMDTs than the version of the model described by Wallaert et al. (2017), which used a single off-frequency gammatone filter centered 1 Cam above the carrier frequency. More precisely, temporal integration of AM for $f_{m}=20 \mathrm{~Hz}$ for NHy listeners was better predicted by the model of Wallaert et al. (2017). This difference resulted partly from differences in the number and center frequencies of the simulated auditory filters and partly from differences in the implementation of the template-matching decision device [a simple correlation process in Wallaert et al. (2017) versus cross-correlation in the current model]. It appears that the cross-correlation device is more efficient than a simple correlation device and real listeners. The cross-correlation device is more robust to (internal) noise than a simple correlation device. The current simulations also suggest that real listeners are less optimal than assumed here. It may be the case that human listeners cannot make perfect comparisons between their internal template and the noisy output of the modulation filters. In future studies, this central inefficiency could be modeled by adding a temporal jitter to the lags used by the cross-correlation process, hence limiting more strongly the comparison between the internal template and the outputs of the modulation filters at high modulation rates.

\section{B. Effects of age and hearing loss on FM detection}

The FMDTs for the HIo listeners were significantly higher (poorer) than those for the NHo listeners. This detrimental effect of hearing loss on FM detection is consistent with previous results obtained for older listeners with sensorineural hearing loss (Moore and Skrodzka, 2002; LacherFougère and Demany, 1998; Buss et al., 2004; Strelcyk and Dau, 2009; Ernst and Moore, 2012; Paraouty et al., 2016) when the NH and $\mathrm{HI}$ groups were compared at equal SLs.

The detrimental effect of age on FM detection predicted by the model depended on a small increase in the additive internal noise used to degrade the internal representation of temporal-envelope cues at the outputs of the modulation filters. The detrimental effect of hearing loss on FM detection predicted by the model depended on the combined effects of 
(1) the broadening of the gammatone filters and the consequent reduction of FM-to-AM conversion and (2) a substantial increase in the additive internal noise. The increase in internal noise had to be substantial, because the detrimental effects were partially counteracted by the loss of cochlear compression and subsequent enhancement of temporalenvelope cues resulting from FM-to-AM conversion.

Although the model reproduced some trends in the data, the model did not provide a unified account of AM and FM detection. The modeling showed that whatever the bandwidth of the simulated auditory filters, the predicted FMDTs for $f_{m}=2 \mathrm{~Hz}$ were higher than obtained (especially for the NHy listeners) and temporal integration of FM was overestimated for both modulation rates for all three groups. Although it is conceivable that more accurate predictions of FMDTs and temporal integration for $f_{m}=20 \mathrm{~Hz}$ may be obtained by using a more accurate simulation of cochlear frequency selectivity and by making the cross-correlation process less optimal (see above), it seems unlikely that such a model would succeed in predicting the low FMDTs found for the NHy listeners for $f_{m}=2 \mathrm{~Hz}$. The general failure of this multi-band model of temporal-envelope processing argues for separate mechanisms for AM and FM detection for very low modulation rates and low carrier frequencies.

\section{Interference effects of AM on FM}

The interaction between the presence/absence of interfering AM and modulation rate was significant, the detrimental effect of the AM being greater for $f_{m}=20 \mathrm{~Hz}$ than for $f_{m}=2 \mathrm{~Hz}$ (Fig. 2), but this effect was mainly driven by the results for the NHy group, as supported by the significant three-way interaction between group, presence/absence of AM, and modulation rate. The results for the NHy group replicate previous findings (Moore and Sek, 1996; Ernst and Moore, 2010, 2012). The results are consistent with the idea that, for the NHy listeners: (1) FM detection depended partly on a cue or cues other than temporal envelope, presumably TFS cues, and that (2) TFS cues were only slightly disrupted by the interfering AM. The ability to use TFS cues worsens with increasing age (Moore et al., 2012a; Moore et al., 2012b) and with hearing loss (Hopkins and Moore, 2007; Moore, 2014) and this may account for the finding that the effect of the interfering AM for $f_{m}=2 \mathrm{~Hz}$ was greater for the NHo and HIo groups than for the NHy group.

Although this explanation is plausible, the model based on temporal-envelope cues alone did predict a greater effect of the interfering AM on FM detection for $f_{m}=20 \mathrm{~Hz}$ than for $f_{m}=2 \mathrm{~Hz}$, when the auditory filters were assumed to have bandwidths of $1 \mathrm{ERB}_{\mathrm{N}}$ for the $\mathrm{NH}$ groups and $2 \mathrm{ERB}_{\mathrm{N}}$ for the HIo group (see Fig. 6, bottom). This happened because, in the model, AM phase information was removed for modulation filters tuned above $10 \mathrm{~Hz}$ but preserved for modulation filters tuned below $2 \mathrm{~Hz}$. This loss of phase information limited the effectiveness of the cross-correlation device (the template-matching process) in extracting the pattern of envelope cues resulting from FM-to-AM conversion for $f_{m}=20 \mathrm{~Hz}$. However, the model may be unrealistic in the assumption that AM phase can be compared precisely across channels for low $f_{m}$ and small AM depths (Green et al., 1990), as is implicit in the template-matching process. In addition, the interference effect predicted by the model for $f_{m}=2 \mathrm{~Hz}$ was much smaller than the obtained interference effect, and the model predicted similar interference effects for all three groups, which is inconsistent with the data. Therefore, it seems that the model does not correctly predict the pattern of interference effects produced by the AM.

Assuming that FM detection for $f_{m}=2 \mathrm{~Hz}$ was mediated by the use of TFS cues, at least for the NHy listeners, the obtained results suggest that interfering AM does have some adverse effect on the ability to use TFS cues. This may occur because the phase at which nerve spikes occur depends somewhat on stimulus level (Johnson, 1980; Palmer and Russell, 1986; Ernst and Moore, 2010) and on whether the amplitude is increasing or decreasing (Moore, 1973). Hence, the AM may lead to small fluctuations in the TFS that resemble those produced by FM, making AM detection harder. It may also be the case that adding the AM to FM makes the task more cognitively challenging. In the absence of AM, listeners merely have to detect that there is some kind of fluctuation at a $2-\mathrm{Hz}$ rate in the target interval. When the interfering AM is present, a strong fluctuation in loudness at a $2-\mathrm{Hz}$ rate occurs in both intervals, and the listener has to identify the interval with the weak additional fluctuation in pitch. This may be more difficult, in the same way that the detection of changes in the pitch of complex tones is more difficult when the tones being compared differ in harmonic content, and therefore timbre (Moore and Glasberg, 1990). Such interference effects are not captured by the current model structure.

\section{Temporal integration of FM cues}

Consistent with the observation of enhanced temporal integration of AM for HIo listeners, the model predicted that temporal integration of FM should be greater for HIo than for NHy and NHo listeners. This trend is visible in Figs. 7 and 8 . The ratio between the predicted FMDTs for $f_{m}=20 \mathrm{~Hz}$ at $N=2$ and $N=9$ was 2 for the HIo model and 1.6 for the NHy model. In fact, the temporal integration of FM information was similar for the three groups. It appears that sensorineural hearing loss does not affect the central processes underlying temporal integration of FM information, such as the combination of "multiple looks" (Viemeister and Wakefield, 1991) or template matching (Dau et al., 1997a,b).

For FM detection in the presence of interfering AM, temporal integration was very small for all three groups (Fig. 3 , bottom panels); FMDTs changed by less than a factor of 2 as $N$ was increased from 2 to 9 . This may have happened because the disruptive effect of the AM depended primarily on the phase of the AM relative to that of the FM in the signal interval (Moore and Sek, 1992); some relative phases are more disruptive than others. For example, when the instantaneous frequency is moving upwards, this leads to a decrease in excitation level on the low-frequency side of the excitation pattern, but this might be cancelled or changed into an increase if the amplitude is also increasing. Although the relative phase of the AM and FM changed from one trial to 
the next, it was fixed within a given stimulus. Essentially, for a stimulus with $N$ cycles, the same information was repeated $N$ times. The "noise" associated with the random variation in the relative phase of the AM and FM may have limited the amount of temporal integration.

Overall, the small amount of temporal integration for FM in the presence of interfering AM was not predicted by the model (Figs. 7 and 8, bottom panels). It may be the case that a template based on a single sample of AM +FM with random relative phase does not represent adequately what the auditory system would do to perform the task. A more realistic simulation might be to use a template based on a sample of FM alone. This strategy was simulated for the FM+AM condition (see Fig. 9, top-right panel) but the predictions of temporal integration for the NHy group were no more accurate than those obtained using an "optimal" template obtained by subtracting the patterns at the outputs of the modulation filters for the target and comparison intervals (see Fig. 9, top-left panel). Thus, as pointed out above, the failure of the model probably occurs because human listeners cannot make perfect comparison between their internal template and the output of modulation filters, or they cannot construct a perfect (or "optimal" template based on the difference between the internal representations of the two intervals.

The data and model predictions were obtained using FM stimuli in the presence of interfering AM with a fixed starting phase only. To assess how the model handled greater stimulus uncertainty, we compared model predictions with and without roving of the phase of the interfering AM. The results are plotted in Fig. 9 (bottom panels) using both types

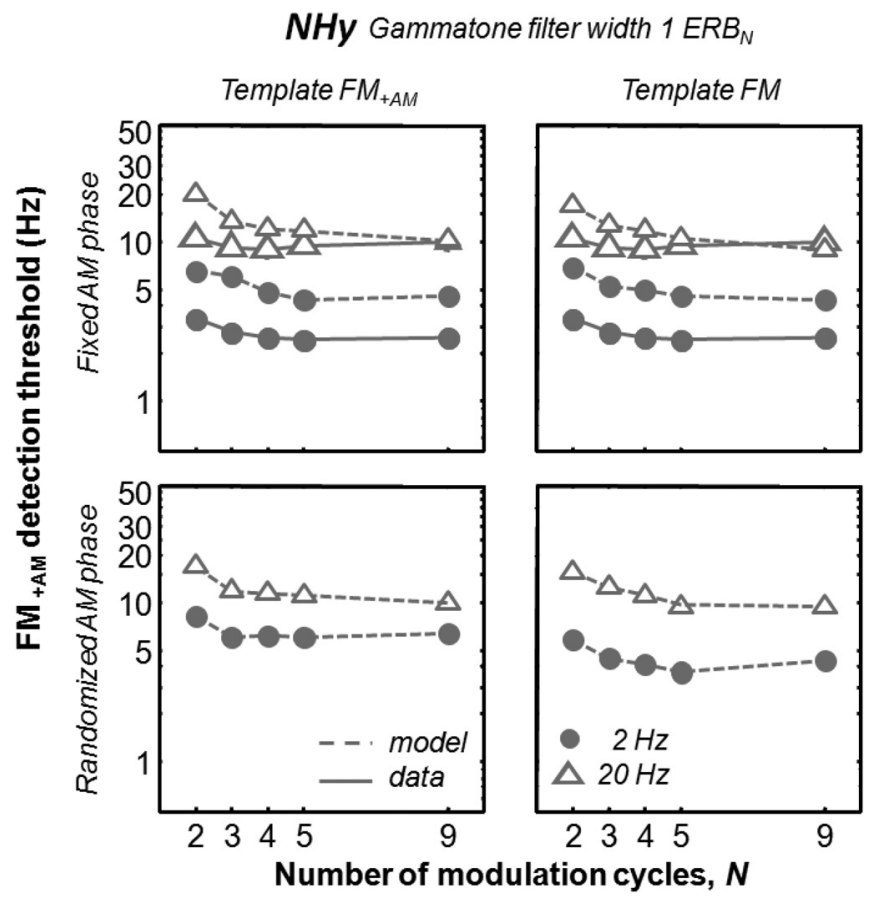

FIG. 9. Predicted FMDTs with interfering AM for the NHy group obtained using either an optimal template based on the difference between the internal representations of each interval (left panels) or a template based on a sample of FM alone (right panels), for a fixed AM phase (top panels) or randomized AM phase (bottom panels). of templates (an "optimal" template vs a sub-optimal template based on a single sample of FM). Similar predictions were obtained with and without roving the AM phase, indicating that the cross-correlation device used at the decision stage of the present model could handle uncertainty as to $\mathrm{AM}$ and FM starting phases, presumably as real listeners would. This suggests that the current model structure is adequate, although real listeners appear less optimal than the model, presumably because their internal templates are not perfect. Further work is warranted to explore to which extent the cross-correlation process is noisy. Two alternative structures of the model could also be considered in future studies of FM detection and integration. The first model, developed by Ewert and Dau (2000), discards temporal-envelope phase information and uses the envelope power as a decision statistic. The second model, developed by Ewert et al. (2018), also discards temporal-envelope phase information and uses both TFS and envelope cues.

\section{SUMMARY AND CONCLUSIONS}

The effects of age and sensorineural hearing loss on the detection and temporal integration of AM and FM were investigated. FMDTs were measured at 40 or $80 \mathrm{~dB}$ SPL (whichever was lower) for a group of listeners with mild-tomoderate sensorineural hearing loss, aged between 50 and 64 years, using a carrier frequency of $500 \mathrm{~Hz}$ and modulation rates of 2 and $20 \mathrm{~Hz}$. The number of modulation cycles, $N$, varied between 2 and 9. The data were compared with FMDTs and AMDTs measured for young and older normalhearing listeners (Wallaert et al., 2016; Wallaert et al., 2017).

FMDTs were higher for the hearing-impaired listeners than for the normal-hearing listeners, but the effect of increasing $N$ was similar across groups. In contrast, AMDTs were lower (better) for the hearing-impaired listeners and the effect of increasing $N$ was greater for them than for the normal-hearing listeners.

A model based on peripheral filtering and envelope extraction followed by a modulation filterbank and a template-based optimal detector was developed to interpret the data. AM and FM detection was limited by two internal noises (an additive noise and an additive memory noise) applied after modulation filtering, whose SD was adjusted to fit the AMDTs. Overall, the model accounted better for FMDTs for the $20-\mathrm{Hz}$ modulation rate than for the $2-\mathrm{Hz}$ rate, and predicted greater temporal integration for FM than observed for all groups.

Taken together, the psychophysical data and modeling results suggest that (1) FM detection for a modulation rate of $2 \mathrm{~Hz}$ and low carrier frequencies probably depends on a cue or cues other than those arising from FM-to-AM conversion, for example cues related to temporal fine structure; (2) greater age is associated with increased FMDTs and this can be explained partly by increased internal noise and partly by reduced sensitivity to temporal fine structure; (3) hearing loss impairs the mechanisms responsible for FM detection, but preserves the memory and decision processes responsible for temporal integration of FM. 


\section{ACKNOWLEDGMENTS}

We thank the listeners for taking part in this study. N.W. was supported by a grant from Neurelec Oticon Medical. C.L. was supported by two grants from ANR (HEARFIN and HEART projects). Brian Moore was supported by the EPSRC (UK, Grant No. RG78536). This work was also supported by Grant Nos. ANR-11-0001-02 PSL* and ANR-10-LABX-0087. We thank two reviewers for helpful comments on an earlier version of this paper.

Ardoint, M., Lorenzi, C., Pressnitzer, D., and Gorea, A. (2008). "Perceptual constancy in the temporal envelope domain," J. Acoust. Soc. Am. 123, 1591-1601.

Bharadwaj, H. M., Masud, S., Mehraei, G., Verhulst, S., and ShinnCunningham, B. G. (2015). "Individual differences reveal correlates of hidden hearing deficits," J. Neurosci. 35, 2161-2172.

Buss, E., Hall, J. W. III, and Grose, J. H. (2004).“Temporal fine-structure cues to speech and pure tone modulation in observers with sensorineural hearing loss," Ear Hear. 25, 242-250.

Carney, L. H., Heinz, M. G., Evilsizer, M. E., Gilkey, R. H., and Colburn, H. S. (2002). "Auditory phase opponency: A temporal model for masked detection at low frequencies," Acta Acust. Acust. 88, 334-346.

Dau, T., Kollmeier, B., and Kohlrausch, A. (1997a). "Modeling auditory processing of amplitude modulation. I. Detection and masking with narrow-band carriers," J. Acoust. Soc. Am. 102, 2892-2905.

Dau, T., Kollmeier, B., and Kohlrausch, A. (1997b). "Modeling auditory processing of amplitude modulation. II. Spectral and temporal integration,” J. Acoust. Soc. Am. 102, 2906-2919.

Davis, K. A., Hancock, K. E., and Delgutte, B. (2010). "Computational models of inferior colliculus neurons," in Computational Models of the Auditory System (Springer, Boston, MA), pp. 129-176.

Demany, L., and Semal, C. (1986). "On the detection of amplitude modulation and frequency modulation at low modulation frequencies," Acta Acust. Acust. 61, 243-255.

Demany, L., and Semal, C. (1989). "Detection thresholds for sinusoidal frequency modulation,” J. Acoust. Soc. Am. 85, 1295-1301.

Emmerich, D. S., Ellermeier, W., and Butensky, B. (1989). "A reexamination of the frequency discrimination of random-amplitude tones, and a test of Henning's modified energy-detector model," J. Acoust. Soc. Am. 85, 1653-1659.

Ernst, S. M. A., and Moore, B. C. J. (2010). "Mechanisms underlying the detection of frequency modulation," J. Acoust. Soc. Am. 128, 3642-3648.

Ernst, S. M. A., and Moore, B. C. J. (2012). "The role of time and place cues in the detection of frequency modulation by hearing-impaired listeners," J. Acoust. Soc. Am. 131, 4722-4731.

Ewert, S. D., and Dau, T. (2000). "Characterizing frequency selectivity for envelope fluctuations," J. Acoust. Soc. Am. 108, 1181-1196.

Ewert, S. D., Paraouty, N., and Lorenzi, C. (2018). "A two-path model of auditory modulation detection using temporal fine structure and envelope cues," Eur. J. Neurosci. (published online).

Ewert, S. D., Verhey, J. L., and Dau, T. (2002). "Spectro-temporal processing in the envelope-frequency domain," J. Acoust. Soc. Am. 112, 2921-2931.

Füllgrabe, C., Moore, B. C. J., and Stone, M. A. (2015). "Age-group differences in speech identification despite matched audiometrically normal hearing: Contributions from auditory temporal processing and cognition," Front. Aging Neurosci. 6, 1-25.

Glasberg, B. R., and Moore, B. C. J. (1986). "Auditory filter shapes in subjects with unilateral and bilateral cochlear impairments," J. Acoust. Soc. Am. 79, 1020-1033.

Glasberg, B. R., and Moore, B. C. J. (1990). "Derivation of auditory filter shapes from notched-noise data," Hear. Res. 47, 103-138.

Goodman, D. F., Winter, I. M., Léger, A. C., de Cheveigné, A., and Lorenzi, C. (2017). "Modelling firing regularity in the ventral cochlear nucleus: Mechanisms, and effects of stimulus level and synaptopathy," Hear. Res. 358, 98-110.

Green, D. M., Richards, V. M., and Onsan, Z. A. (1990). "Sensitivity to envelope coherence," J. Acoust. Soc. Am. 87, 323-329.

Hartmann, W. M., and Klein, M. A. (1980). "Theory of frequency modulation detection for low modulation frequencies," J. Acoust. Soc. Am. 67, 935-946.
He, N., Mills, J. H., and Dubno, J. R. (2007). "Frequency modulation detection: Effects of age, psychophysical method, and modulation waveform," J. Acoust. Soc. Am. 122, 467-477.

Henry, K. S., Kale, S., and Heinz, M. G. (2016). "Distorted tonotopic coding of temporal envelope and fine structure with noise-induced hearing loss," J. Neurosci. 36, 2227-2237.

Hopkins, K., and Moore, B. C. J. (2007). "Moderate cochlear hearing loss leads to a reduced ability to use temporal fine structure information," J. Acoust. Soc. Am. 122, 1055-1068.

Jerger, J. (1962). "The SISI test," Int. Audiol. 1, 246-247.

Johannesen, P. T., Pérez-González, P., Kalluri, S., Blanco, J. L., and LopezPoveda, E. A. (2016). "The influence of cochlear mechanical dysfunction, temporal processing deficits, and age on the intelligibility of audible speech in noise for hearing-impaired listeners," Trends Hear. 20, 1-14.

Johnson, D. H. (1980). "The relationship between spike rate and synchrony in responses of auditory-nerve fibers to single tones," J. Acoust. Soc. Am. 68, 1115-1122.

Kortlang, S., Mauermann, M., and Ewert, S. D. (2016). "Suprathreshold auditory processing deficits in noise: Effects of hearing loss and age," Hear. Res. 331, 27-40.

Kujawa, S. G., and Liberman, M. C. (2009). "Adding insult to injury: Cochlear nerve degeneration after 'temporary' noise-induced hearing loss," J. Neurosci. 29, 14077-14085.

Lacher-Fougère, S., and Demany, L. (1998). "Modulation detection by normal and hearing-impaired listeners," Audiology 37, 109-121.

Levitt, H. (1971). "Transformed up-down methods in psychoacoustics," J. Acoust. Soc. Am. 49, 467-477.

Lorenzi, C., Soares, C., and Vonner, T. (2001). "Second-order temporal modulation transfer functions," J. Acoust. Soc. Am. 110, 1030-1038.

Lutman, M. E., Gatehouse, S., and Worthington, A. G. (1991). "Frequency resolution as a function of hearing threshold level and age," J. Acoust. Soc. Am. 89, 320-328.

Maiwald, D. (1967). "Die berechnung von modulationsschwellen mit hilfe eines funktionsschemas" ("Calculation of differential thresholds by means of a functional model"), Acustica 18, 193-207.

Moore, B. C. J. (1973). "Frequency difference limens for narrow bands of noise," J. Acoust. Soc. Am. 54, 888-896.

Moore, B. C. J. (2007). Cochlear Hearing Loss: Physiological, Psychological and Technical Issues (Wiley, Chichester, UK), pp. 1-332.

Moore, B. C. J. (2008). "The role of temporal fine structure processing in pitch perception, masking, and speech perception for normal-hearing and hearing-impaired people," J. Assoc. Res. Otolaryngol. 9, 399-406.

Moore, B. C. J. (2014). Auditory processing of temporal fine structure: Effects of age and hearing loss (World Scientific, Singapore), pp. 1-182.

Moore, B. C. J., Füllgrabe, C., and Sek, A. (2009). "Estimation of the center frequency of the highest modulation filter," J. Acoust. Soc. Am. 125, 1075-1081.

Moore, B. C. J., and Glasberg, B. R. (1989). "Mechanisms underlying the frequency discrimination of pulsed tones and the detection of frequency modulation," J. Acoust. Soc. Am. 86, 1722-1732.

Moore, B. C. J., and Glasberg, B. R. (1990). "Frequency discrimination of complex tones with overlapping and non-overlapping harmonics," J. Acoust. Soc. Am. 87, 2163-2177.

Moore, B. C. J., Glasberg, B. R., Stoev, M., Füllgrabe, C., and Hopkins, K. (2012a). "The influence of age and high-frequency hearing loss on sensitivity to temporal fine structure at low frequencies," J. Acoust. Soc. Am. 131, 1003-1006.

Moore, B. C. J., and Sek, A. (1992). "Detection of combined frequency and amplitude modulation,” J. Acoust. Soc. Am. 92, 3119-3131.

Moore, B. C. J., and Sek, A. (1994). "Effects of carrier frequency and background noise on the detection of mixed modulation," J. Acoust. Soc. Am. 96, 741-751.

Moore, B. C. J., and Sek, A. (1996). "Detection of frequency modulation at low modulation rates: Evidence for a mechanism based on phase locking," J. Acoust. Soc. Am. 100, 2320-2331.

Moore, B. C. J., and Skrodzka, E. (2002). "Detection of frequency modulation by hearing-impaired listeners: Effects of carrier frequency, modulation rate, and added amplitude modulation," J. Acoust. Soc. Am. 111, 327-335.

Moore, B. C. J., Vickers, D. A., and Mehta, A. (2012b). "The effects of age on temporal fine structure sensitivity in monaural and binaural conditions," Int. J. Audiol. 51, 715-721.

Oxenham, A. J., and Shera, C. A. (2003). "Estimates of human cochlear tuning at low levels using forward and simultaneous masking," J. Assoc. Res. Otolaryngol. 4, 541-554. 
Palmer, A. R., and Russell, I. J. (1986). "Phase-locking in the cochlear nerve of the guinea-pig and its relation to the receptor potential of inner haircells," Hear. Res 24, 1-15.

Paraouty, N., Ewert, S. D., Wallaert, N., and Lorenzi, C. (2016). "Interactions between amplitude modulation and frequency modulation processing: Effects of age and hearing loss," J. Acoust. Soc. Am. 140, $121-131$.

Paraouty, N., Stasiak, A., Lorenzi, C., Varnet, L., and Winter, I. M. (2018). "Dual coding of frequency modulation in the ventral cochlear nucleus," J. Neurosci. 38, 4123-4137.

Patterson, R. D., Allerhand, M. H., and Giguère, C. (1995). "Time-domain modeling of peripheral auditory processing: A modular architecture and a software platform," J. Acoust. Soc. Am. 98, 1890-1894.

Paul, B. T., Bruce, I. C., and Roberts, L. E. (2017). "Evidence that hidden hearing loss underlies amplitude modulation encoding deficits in individuals with and without tinnitus," Hear. Res. 344, 170-182.

Peters, R. W., and Moore, B. C. J. (1992). "Auditory filters and aging: Filters when auditory thresholds are normal," in Auditory Physiology and Perception, edited by Y. Cazals, L. Demany, and K. Horner (Pergamon, Oxford), pp. 179-185.

Rees, A., and Malmierca, M. S. (2005). "Processing of dynamic spectral properties of sounds," Int. Rev. Neurobiol. 70, 299-330.

Robles, L., and Ruggero, M. A. (2001). "Mechanics of the mammalian cochlea," Physiol. Rev. 81, 1305-1352.

Ruggero, M. A. (1994). "Cochlear delays and traveling waves: Comments on 'Experimental look at cochlear mechanics': [A. Dancer, Audiology 1992;31: 301-312]," Audiology 33, 131-142.

Saberi, K., and Hafter, E. R. (1995). "A common neural code for frequencyand amplitude-modulated sounds," Nature 374, 537-539.

Schoof, T., and Rosen, S. (2014). "The role of auditory and cognitive factors in understanding speech in noise by normal-hearing older listeners," Front. Aging Neurosci. 6, 307.

Sek, A., and Moore, B. C. J. (1995). "Frequency discrimination as a function of frequency, measured in several ways," J. Acoust. Soc. Am. 97, 2479-2486.

Sek, A., and Moore, B. C. J. (2002). "Mechanisms of modulation gap detection," J. Acoust. Soc. Am. 111, 2783-2792.

Shamma, S. A. (1985). "Speech processing in the auditory system II: Lateral inhibition and the central processing of speech evoked activity in the auditory nerve," J. Acoust. Soc. Am. 78, 1622-1632.
Shamma, S. A., and Lorenzi, C. (2013). "On the balance of envelope and temporal fine structure in the encoding of speech in the early auditory system," J. Acoust. Soc. Am. 133, 2818-2833.

Sheft, S., Shafiro, V., Lorenzi, C., McMullen, R., and Farrell, C. (2012). "Effects of age and hearing loss on the relationship between discrimination of stochastic frequency modulation and speech perception," Ear Hear. 33, 709-720.

Sheft, S., and Yost, W. A. (1990). "Temporal integration in amplitude modulation detection,” J. Acoust. Soc. Am. 88, 796-805.

Strelcyk, O., and Dau, T. (2009). "Relations between frequency selectivity, temporal fine-structure processing, and speech reception in impaired hearing," J. Acoust. Soc. Am. 125, 3328-3345.

Tchorz, J., and Kollmeier, B. (1999). "A model of auditory perception as front end for automatic speech recognition," J. Acoust. Soc. Am. 106, 2040-2050.

Verschuure, J., and van Meeteren, A. A. (1975). "The effect of intensity on pitch," Acustica 32, 33-44.

Viemeister, N. F. (1979). "Temporal modulation transfer functions based upon modulation thresholds," J. Acoust. Soc. Am. 66, 1364-1380.

Viemeister, N. F., and Wakefield, G. H. (1991). "Temporal integration and multiple looks," J. Acoust. Soc. Am. 90, 858-865.

Wallaert, N., Moore, B. C. J., Ewert, S. D., and Lorenzi, C. (2017). "Sensorineural hearing loss enhances auditory sensitivity and temporal integration for amplitude modulation,” J. Acoust. Soc. Am. 141, 971-980.

Wallaert, N., Moore, B. C. J., and Lorenzi, C. (2016). "Comparing the effects of age on amplitude modulation and frequency modulation detection," J. Acoust. Soc. Am. 139, 3088-3096.

Whiteford, K. L., Kreft, H. A., and Oxenham, A. J. (2017). “Assessing the role of place and timing cues in coding frequency and amplitude modulation as a function of age," J. Assoc. Res. Otolaryngol. 18, 619-633.

Zeng, F.-G., Nie, K., Stickney, G. S., Kong, Y.-Y., Vongphoe, M., Bhargave, A., and Cao, K. (2005). "Speech recognition with amplitude and frequency modulations,” Proc. Natl. Acad. Sci. U.S.A. 102, 2293-2298.

Zwicker, E. (1952). "Die Grenzen der Hörbarkeit der Amplitudenmodulation und der Frequenzmodulation eines Tones" ("The limits of audibility of amplitude modulation and frequency modulation of a pure tone"), Acustica 2, 125-133.

Zwicker, E. (1956). "Die elementaren Grundlagen zur Bestimmung der Informationskapazität des Gehörs" ("The foundations for determining the information capacity of the auditory system"), Acustica 6, 356-381. 